\title{
IEEE Transactions on Geoscience and Remote Sensing
}

\section{Singularity Power Spectra: A method to assess geophysical consistency of gridded products. Application to Sea Surface Salinity remote sensing maps.}

\author{
Hoareau, N., A. Turiel, M. Portabella, J. Ballabrera, and J. \\ Vogelzang
}

Dear editor,

We want to thank you and the three reviewers, whose comments and suggestions are definitely contributing to improve the readability and scientific quality of this manuscript. In response, we have made an extensive revision of our paper to respond all the questions of the reviewers.

Sincerely,

Nina Hoareau

\section{Reviewer \#1}

I have reviewed the manuscript entitled "Singularity Power Spectra: A method to assess geophysical consistency of gridded products. Application to Sea Surface Salinity remote sensing maps" by Hoareau and co-authors, submitted to Transactions on Geoscience and Remote Sensing. The authors 
apply a new method based on singularity analysis and singularity spectral analysis to assess the geophysical properties among different sea surface salinity (SSS) gridded datasets (satellite; in-situ derived climatologies; model). The significant point of the paper is that beyond the sources of uncertainty in remote sensing SSS products, we can extract valuable dynamical information from them, providing a tool for evaluating different products and feedback for product improvement. My main suggestion is related to the satellite SSS products used. There is a variety of SMOS SSS products generated by different institutions (e.g. Ifremer and even an OI version) and for the scientific community is hard to be able to identify which product to use. I think this kind of analysis would be useful applied to other comparable products as well. For this analysis the authors are using the latest SMOS BEC products (binned (L3), optimally interpolated and Level 4 maps) and an earlier version (V3) of the Aquarius and related CAP version as well. It is not clear to me which product of the mapped V3 are using and that should be clarified and why to use and older version of it. Aquarius V4 (and CAP version) had been available for a while now, and there is also and OI product available as well (all available from PO.DAAC). Aquarius V4 made significant corrections over V3. It would be valuable if the authors could use the most recent versions within their analysis and incorporate the results in the manuscript. You could replace V3 by V4, or at least mention in the text if the results of the analysis are not significantly different.

We have replaced Aquarius V3 by V4 throughout the manuscript (note though that both V3 and V4 yield very similar results). The novel SPS approach presented in this paper is applied to a wide variety of datasets, but can of course be applied to any source of SSS (or any other geophysical) data, including Ifremer's and other available satellite SSS products. This first paper mainly aims at proving the usefulness of the proposed methodology for assessing the geophysical consistency of SSS maps. A sentence has been added in the concluding section regarding the method's wider potential: "Moreover, the proposed SPS methodology is generic and can be applied to assess the consistency of any geophysical ocean product/map."

I personally believe it would be useful to assess the scales of the geophysical 
structures among the latest versions of the available products (SMOS BEC, Aquarius and Ifremer, and their interpolated versions).

We have added the following paragraph in the conclusion:

With the help of the new singularity spectral analysis, it can be concluded that: i) the SPS of SL3 shows excessive variance and slopes that are the most incompatible with the rest of the products; ii) the processing updates used to generate S13 over S09 are justified from the point of view of the spatial spectra; iii) the large scale variability of the rest of the products are equivalent (scales larger than $800 \mathrm{~km}$ ); iv) below $800 \mathrm{~km}$, the slope of the CORA salinity product (SC) becomes too steep in some regions; v) below $400 \mathrm{~km}$, only the satellite and the model output surface salinities share a slope similar to that of TO; and finally, vi) for shorter scales (below $200 \mathrm{~km}$ ), and despite the fact that no product has the same slope as the reference temperature field, the SL4 remains the product whose slope is the closest to that reference.

\section{Minor comments:}

Figures:

On some of the figures the fonts are already too small, please adjust accordingly to the final size of the figure. On the multi-panel figures such as 2,3 and 5 please include the labels for each case as you did in figure 9. I understand you will have in the caption but it is much easier for the reader if you just label the figures; you could then remove it from the captions if you already explained on the main text and expanded on one of the captions.

Done

Text:

References: there are some discrepancies among some of the cited references within the text and with its version in the reference list. I'm including some of them. Please check them all. 


\section{Minor comments:}

Page 6 line 47: OSTIA SST, first time mentioning the product, expand and reference.

Definition of acronym and reference added.

Specify the version the Aquarius product used and include its references (e.g. Section 2.1.2)

Done.

Page7 line57: “Turiel et al [2008]”. Is it a or b?

2008b, now corrected.

Missing from the reference list: page 7 line 31: "Jorda et al."

Included, thanks.

Page 9 line 25: "Parent et al. [2013]”. In the reference list is cited as 2012.

Modified, thanks.

Page 9 line 10: CORA3.3 (please include reference).

Added reference Cabanes et al., (2013): The CORA dataset: validation and diagnostics of in-situ ocean temperature and salinity measurements. Ocean Sci., 9, 1-18, 2013.

Page 9 line 48: “(INSITU_GLO...”, please expand or reference.

The INSITU_GLO_TS_REP_OBSERVATIONS_013_001_b refers to the in situ data set compiled by Copernicus and used for the optimal interpolation; as its reference is not strictly necessary and to avoid introducing references, we have remove the name of the data set.

Page 10 line 9: "Gaillard et al. 2016”. Is it 2016 or 2015? 
Modified, thanks.

Page 10 line 55 to 60: OSTIA reference: move those lines to page 6 line 47.

Modified.

Page 21 line 35: "Press et al. 1986". Discrepancy with citation in the reference list.

Modified, thanks.

Page 21 line 54-55: "[Emery and Thomson, 2001]" replace by Emery and Thomson [2001].

Done.

Page 23 line 54: "products is able" replace by products are able.

Done.

Also, I disagree with the statement "none of the products is able to reproduce..." since I only see the problem for figures 8: $e$, f and $i$.

Indeed, in Figures 9e, 9f and 9i the SPS are truncated at $200 \mathrm{~km}$ because S09, $\mathrm{Aq}$, and $\mathrm{AqC}$ have a resolution of 1 degree. However, we were referring to the fact that the slopes below $200 \mathrm{~km}$ are actually steeper than -2.4. This has been clarified as follows: "Comparing the SPS slopes of the products (Figure 9) below $200 \mathrm{~km}$ with those of TO (all about -2.4, as seen in Figure 8), none of the SSS products consistently reproduce the reference turbulent cascade below $200 \mathrm{~km}$ for all regions, including the numerical simulation (SG)."

\section{Reviewer \#2}

The paper is well written. 
Main points of concern:

1-The authors consider the SE map corresponding to OSTIA SST as the SE map reference. This choice should be more discussed. The SE map from OSTIA products could be compared to SE map derived from SST CORA products and/or from an other available SST OI product. The SE conditional PDF between CORA SST and OSTIA SST could be considered. In the same line, the SE conditional PDF between CORA SSS and L3 and L4 SSS products shall be considered and commented.

In fact, as it has been observed in several previous works [Isern-Fontanet, 2007; Nieves, 2008; Umbert, 2014], the singularity exponents of different ocean scalars do correspond, to the extent given by a) the numerical uncertainty of the numerical method used to derive them in practice; $b$ ) the effect of noise; and c) the eventual presence of terms in the evolution equation of the scalar other than the advective derivative capable to spawn singularities. In the works commented above, it was observed that the singularities coming from two different scalars do correspond, for the cases of: remote sensing maps of SST and infrared brightness temperatures [Isern-Fontanet et al, 2007]; remote sensing SST and chlorophyll maps [Nieves et al, 2007]; and output SSS and SSS from a numerical model [Umbert el al, 2014].

We have taken the SE of OSTIA SST as a reference or ground truth because, as discussed in [Turiel, 2008a] and other works, it provides in practice the highest quality set of singularity exponents of all remote sensing products. This is not to say that this reference is perfect, of course it is not, but is much less affected by noise than other remote sensing products and in particular SSS products (this is evidenced by the fine, precise singularity lines in the OSTIA SST exponents).

An example of conditioned histogram is shown by [Umbert et al., 2014], using the Earth Simulator SSS and SST output. See in the Figure below the good correspondence between hSSS and hSST, while singularity independence only emerges by the end of the considered range (-0.5 to 0.5$)$." 


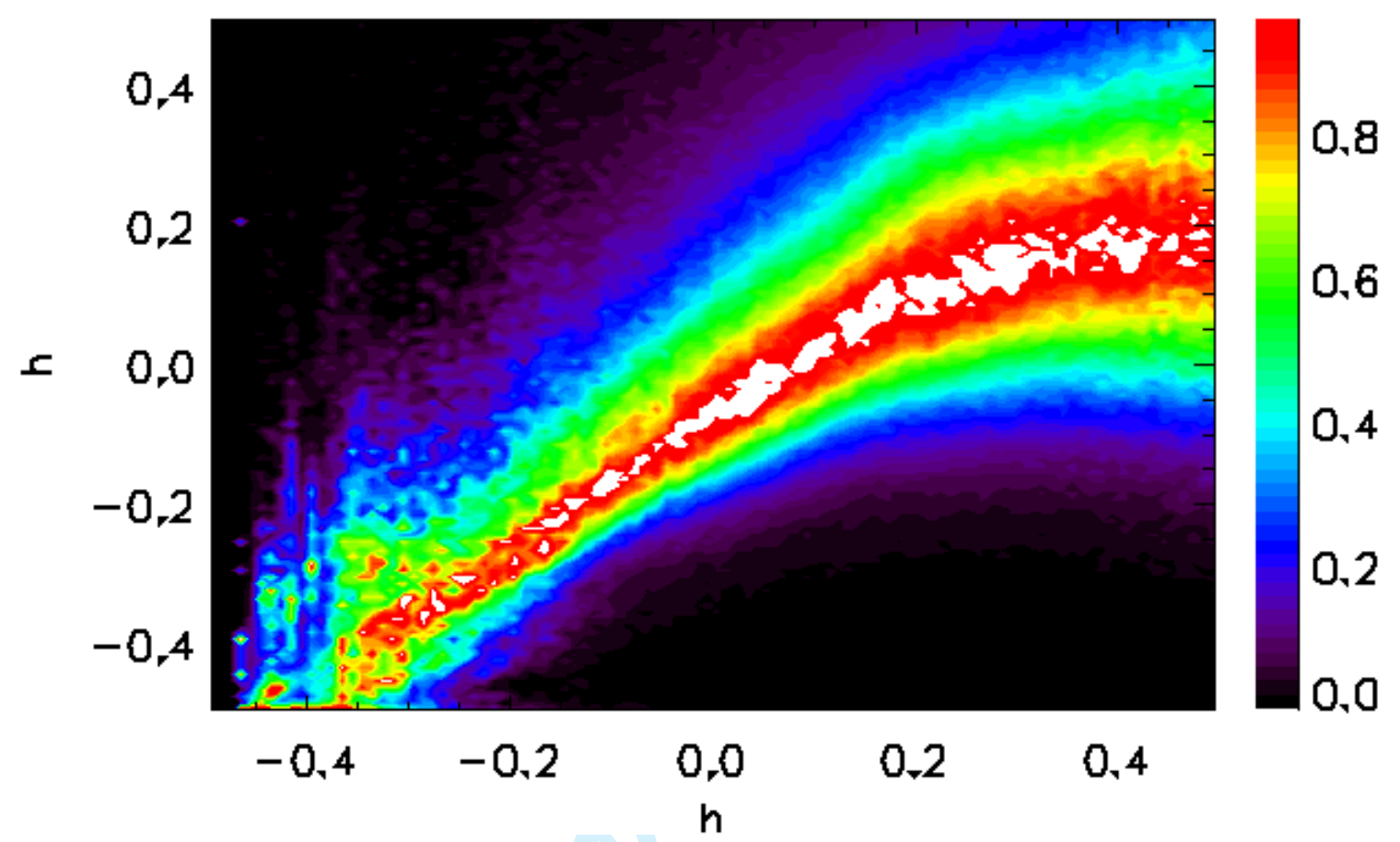

No reference is perfect, but introducing a lengthy discussion about the specific issues of each SSS and SST dataset and its limitations to serve as singularity analysis templates is in our opinion beyond the scope of this paper and probably deserving a paper on its own. Therefore, we have considered more convenient to settle our reference to OSTIA SST (as the highest quality data source) and refer to the papers in which the goodness of such a reference has already been discussed.

2-"the singularity exponents from different ocean variables should be the same" could necessitate a little more attention. It is likely not always the case, or at least can apply to a certain range of scales under a certain dynamical regime. As the SE will easily trace frontal regions, there is no reason to have a perfect alignment between tracer fields: if you consider the existence of a cross-flow mixing from a small-scale process, there will be a persistent misalignment of tracer and flow. Moreover, if the mixing process is different between SST and SSS, then tracers might misalign, and will not have the same 
local regularity. Furthermore, locally fronts can be compensated (to minimize the density change) to possibly blur the tracer regularity. A more in-depth discussion is necessary, especially to better interpret the observed transition obtained from the conditional SE PDF.

By construction, SE are scale invariant, so in the range of scales for which the structure functions present power-law scaling the SE as observed at the different scales coincide (this is discussed in some depth in [Turiel et al, 2008b]. Singularity fronts do not coincide with the isolines of the scalar from which are derived because they are caused by the non-linear character of the advective derivative and are thus everywhere aligned with the streamlines of the flow but not with the isolines (see Figure 9 in [Turiel et al, 2009], reproduced below).
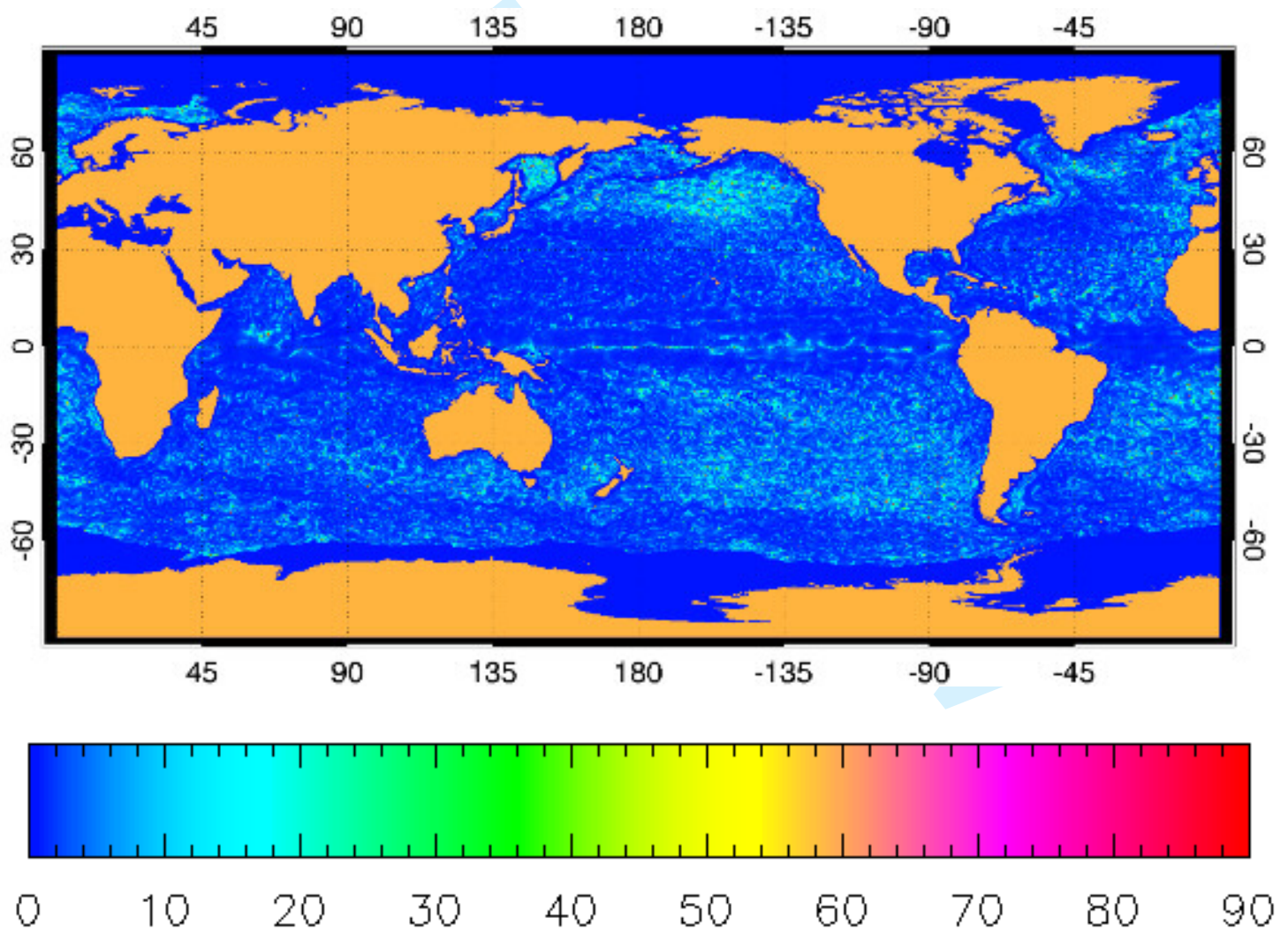

Figure 9 (from [Turiel et al, 2009]): Deviation angle between streamlines and scalar isolines. Top: Deviation angles averaged between the days 1 to 3 July 2003 for the singularity maps (spatial average: $3.4^{\circ}$ ). Bottom: Deviation angles averaged between the days 1 to 3 July 2003 for SST (spatial average: $22.5^{\circ}$ ). 
Certainly, at fine scales, other non-linear processes may change the value of SE and thus produce misalignment between streamlines (i.e., implying misalignment between singularity fronts of SST and SSS), but this has not been shown to be the case at the scales considered in this paper. The following clarifying comment has been introduced in the text:

"In fact, as shown in Figure 9 of [Turiel et al., 2009], singularity fronts derived from OSTIA SST align very well with the streamlines of the flow, so we consider OSTIA SST SE a good reference at the scales considered in this paper".

3-As found, the SPS spectral slopes are about -2.4, corresponding to an SE map spectral slope of about -.4. This is close to noise spectrum. Can the authors comment on the SE error estimates? Is the estimated error of SE map not too depending upon the local SE (regularity)? As fields are certainly very smooth, the derived SE can certainly more strongly impacted. Likely, there is less difficulties to derive local SE over frontal regions. Can the authors comment about it?

We believe that the reviewer means that the SE spectrum is close to that of white noise. When looking at the maps of singularity exponents, one can clearly see that they are far from white noise (white noise means that all the points are random and independent, while the singularity exponents are structured along well defined lines that, not coincidentally, agree with the patterns of ocean circulation). The essential difference is that white noise corresponds to independent points, while points with negative SEs are close to being locally decorrelated but not independent (the dependence is along lines, but in 2D lines are of zero measure - they have zero area - and so they can be considered almost independent when following all directions but that of the singularity front).

Regarding the error in the estimation of SE, as commented in Turiel et al, 
$2008 \mathrm{~b}$, it is known to typically be +-0.05 with the method used in this paper, with a slight tendency to degrade as the value of the SE increases.

Regularizing the signal has no effect on the value of SE, indistinctly larger and smaller, just on the resolution of the map of SE. The application of linear filters on the analyzed signal have no effect on the singularity exponents, as they produce long-range effects (result of convolving with a function) and just change the effective resolution of the data (essentially, the singularity fronts appear to be wider, as corresponds to the actual resolution of the data). Linear filters will damp the SPS at large wavenumbers, as actually observed. Therefore, any regular field obtained using a linear filter (a convolution) would lead to a premature fall of the SPS curve at high wavenumbers but the SEs will be the same as they correspond to its actual (not nominal) resolution. This applies to any value of singularity exponent, so points with larger values of SE are not particularly affected. This is commented in the paper now as follows:

"Singularity exponents are estimated by calculating the slope of a log-log regression of wavelet projections versus wavelet scale [Turiel et al., 2008b]. The wavelet projection of any map regularized by a convolution kernel will be similar as having projected the signal on the wavelet basis at larger scales, adding the characteristic scale of the convolution kernel to the wavelet scale. Therefore, the resulting map of singularity exponents will be of lower resolution (evidenced by thicker singularity lines), and quite naturally would show up as a premature damping of the power spectra at high wavenumbers."

4-Can the conclusion be more focused to help the users about the choice/limitation of the different available products.

We have included the following paragraph in the conclusion:

"With the help of the new singularity spectral analysis, it can be concluded that: i) the SPS of SL3 shows excessive variance and slopes that are the most incompatible with the rest of the products; ii) the processing updates used to generate S13 over S09 are justified from the point of view of the spatial spectra; iii) the large scale variability of the rest of the products are equivalent 
(scales larger than $800 \mathrm{~km}$ ); iv) below $800 \mathrm{~km}$, the slope of the CORA salinity product (SC) becomes too steep in some regions; v) below $400 \mathrm{~km}$, only the satellite and the model output surface salinities share a slope similar to that of TO; and finally, vi) for shorter scales (below $200 \mathrm{~km}$ ), and despite the fact that no product has the same slope as the reference temperature field, the SL4 remains the product whose slope is the closest to that reference."

Reviewer \#3

This paper discusses a new methodology, singularity power spectra, for evaluating the consistency with which a parameter's data in a given product represents a continuous cascade across fluid dynamic scales. The slope and standard deviation of slope for selected representative regions, as well as consistency across the regions, are established as metrics of product quality.

I recommend that this paper be accepted with minor revisions. Comments on content are below. The following notation is used to reference page (P\#) and line (L\#) numbers: P\#L\#.

Extensive, but minor grammar and suggested style edits provided in attached "track-change" version of the paper

\section{Comments}

- General

o Ensure that acronyms are defined when first used, both in the abstract and within the body of the article.

The acronyms have been defined, thanks.

o All figures should have a figure title.o Inconsistent formatting within the Reference section. Check IEEE style manual. Quotations for article title Volume and page numbers Publication date 
Modified.

o For the most part, figure captions should be understandable when standing alone, without drawing upon acronyms defined elsewhere.

Modified.

o Figures are too small for the reader to assess, particularly for those figures with numerous subplots.Recommend identifying/titling each subplot so that the reader can readily identify which analysis is which without having to constantly refer to the caption.

The figures have been modified.

\section{- Title}

o Delete periods

Done

o Consider the following proposed title, "Singularity Power Spectra: A method to assess geophysical consistency of gridded products - Application to sea-surface salinity remote sensing maps"

Thank you, we have removed the dot and written sea surface salinity in lower case. Even if we agree that the hyphen between sea and surface is formally the most correct way to write it, we have decided to keep it as two words since this is the usual convention and widely used within the community. Ultimately, the Editor can decide in the proof reading stage about this.

\section{- Abstract}

-1. Introduction

- P3L70 - Perhaps a brief explanation/description of what is meant by "higher-level processing data processing" could be included for 
readers not familiar with satellite data processing levels.

Modified.

○ P4L103 - "structure of the variable under analysis"

- Perhaps, features identified (or defined) by the parameter being analyzed"?

The sentence has been modified as follows: "... and how the cascade affects the spatial variability of the field being analyzed."

- The term "variable" is used throughout the paper. As used, the term "parameter" is more correct.

As commonly referred to within the remote sensing community, we use indistinctively the words "parameter" and "variable" to refer to geophysical magnitude (temperature, salinity).

\section{-2. Data and regions}

o P6L159 - "Practical Salinity Scale-78” needs the following reference.

- UNESCO, "The Practical Salinity Scale 1978 and the International Equation of State of Seawater 1980," United Nations Educational, Scientific and Cultural Organization, Paris, France, UNESCO Technical Papers in Marine Science Number 36, 10th report of the Joint Panel on Oceanographic Tables and Standards, 1981. Added.

- Perhaps could/should use PSS or PSS-1978 when identifying salinity values.

We have omitted the reference to the practical salinity scale, as there is no possible confusion in this manuscript.

o P7L166 - Using the same acronym (L3) when referencing a specific SMOS product and when discussing general Level-3 processing adds to ready confusion as to the authors'intention. Recommend using a different notation for the SMOS Level-3 product, or using "Level-3," rather than "L3" when discussing generic Level-3 processing.

- Similarly, remove the ambiguity between the SMOS "OI" product and the acronym for the general terminology of "optimal interpolation." 
We have changed the acronyms L3, OI and L4 by SL3, SOI and SL4, respèctively.

o P7L166 - SMOS L3 data set

- Data set reference?

The SMOS SSS products used here correspond to the operational V1.0 generated by the SMOS Barcelona Expert Center (BEC) at 0.25-degree resolution (available at http://cp34-bec.cmima.csic.es). This is now clarified in the text.

- How binned?

As stated in the text, it's a bin average.

- Period of data record used/referenced?

Since we use the same period for all the products, the period is specified in section 2.2

o P7L175 - "SMOS L3 9-day SSS data are optimally interpolated to produce maps with higher spatial and temporal consistency"

- Does the SMOS OI product actually start with already-gridded Level-3 data in its optimal interpolation scheme? It seems that ungridded Level-2 swath data would be optimally interpolated to produce the OI Level-3 gridded data; otherwise, the errors/uncertainties of the "L3" gridding methodology are imbedded in the OA analysis.

Yes, it starts with the already-gridded Level-3 data and our algorithm assumes that the large source of uncertainties of the input data is the variance in each gridded bin.

- Data set reference?

Same as for the SMOS Level-3 binned.

- OI methodology/reference?

The reference, Bretherton et al., (1976) has been added.

- Period of data record used/referenced?

Since we use the same period for all the products, the period is specified in section 2.2 
o P7L177 - Citation for WOA 2009 incorrect. Should be "(Antonov et al., 2009)" per the reference list.

- Conflating reference citation with acronym (WOA 2009) Corrected.

- Establish acronym WOA 2009 Corrected.

o P7L180 - SMOS L4 fused (L4) product

- Data set reference?

Same as for the SMOS Level-3 binned.

- Period of data record used/referenced?

Since we use the same period for all the products, the period is specified in section 2.2

- L4 methodology/reference?

As stated in the text, the data fusion algorithm described by Umbert et al. [2014] is used at BEC to build the SL4 product.

o P7L181 - "the SSS L3 maps are fused with 9-day OSTIA SST maps"

- In what manner are the SSS and SST maps fused for the "L4" product? Density? Singularity analysis?

Due to the length of this manuscript, a description of the fusion algorithm is omitted and we refer to Umbert et al. (2014). In short, the fusion algorithm is a weighted, spatial, local regression between the OSTIA SST and the SMOS L3 SSS.

o P8L201 - “Two Aquarius SSS monthly products ....”

- For format consistency with the SMOS products and for readability, bulletize the two Aquarius products as was done for the three SMOS products.

Done, thank you very much. 
o P10L254 - Revise subsection title. Perhaps, "Regions and time period"

Changed.

o P1OL258 - “NATL: $0^{\circ}-64^{\circ} \mathrm{N}, 27^{\circ}-33^{\circ} \mathrm{W} "$

- For precision, except for the equator, all coordinates should be identified as $N / S$ or $E / W$.

- $27^{\circ}-33^{\circ} \mathrm{W}$ could be either $27^{\circ} \mathrm{E}$ or $27^{\circ} \mathrm{W}$

- This issue occurs throughout Section 2.3

Corrected.

o P11L277 - The material from this point through the remainder of this section belongs in "Section 3. Singularity analysis."

Since Figs. 2 and 3 do not refer to Singularity Analysis, we have created a section 2.4 to analyze the mean and the standard deviation of the SSS products for 2012 .

o P11L288 - With respect to the La Niña signal in Figure 3:

- The signal is not particularly obvious, especially in Figure 3c. Indeed, it has been reported that the SMOS L4 product tends to smooth the salinity field. The following sentence has been added: "As already indicated by Umbert et al. (2014), the fusion algorithm results in a smaller dynamic range of the fused field, e.g. SL4 (Figures 3c)."

- Which feature( $(s)$ are being considered?

We highlight the region of interest with white box in Fig. $3 \mathrm{~h}$

- The figures are small, so it is difficult to discern details.

We have enlarged the figures as much as possible.

o P12L292 - "The variance maps show similar variability levels in the Aquarius (Figures $3 f$ and 3i) and SMOS higher levels OI and L4"

- The size of the figures makes this statement difficult to confirm. L4 appears notably weaker, rather than similar, even with respect to the SMOS L3 and OI plots. 
You are right. See the answer to previous point.

- The Gulf of Tehuantepec region shows a fair bit of variability. Thank you, we have added it.

\section{Singularity analysis}

o P13L339 - "large- and small-scale features of the global circulation, such as the Amazon plume"

- An Amazon plume is not seen in Figure 4 Indeed, the reference to the Amazon has been removed.

- The readability of this figure might be improved by plotting the data as dark on a white background.

We have tried in white and black, but a dark background and white (bright) singularity fronts are actually easier to interpret.

o P14L352 - “The S09 singularity map only shows a few large-scale patterns"

- In looking at Figure 5.e, I do not concur with this characterization. S09 has more than a few large-scale patterns. Perhaps what is meant is that S09 displays only large-scale patterns?

Yes, you are right, corrected.

o P14L358 - "Finally, the singularity map of SG (Figure 5h) reproduces all the streamlines, at all scales, with a similar quality to that of the TO singularity map (Figure 4).”

- Figure 5 h (SG) does not appear significantly different from Figure 5.d (S13), yet SG is characterized as reproducing all the streamlines at all scales?

Note that in contrast with Figure 5d (S13), in Figure 5h (SG) one can see very well organized zonally-oriented streamlines in tropical regions.

\section{-4. Geophysical consistency between SST and SSS}




\begin{abstract}
o P16L397 - "The analysis will be limited to the range of negative singularity exponents of SST"
\end{abstract}

- Figure 6 plots for L4, Aq, AqC, SC, and SG appear to show skill/correspondence up to hSST (OSTIA) $\sim+0.25$, nearly doubling the range of relevance?

While it is true that the one-to-one line of some histograms extend above zero, we take zero as reference since all the histograms show singularity correspondence below zero.

o P16L403 - "meaning that they are more accurate than the other SSS products"

- How is "accuracy" being defined? Does accuracy equate to tight correlation within the specified narrow range? At higher hSST values, the OI and L4 plots appear to have low standard deviation, but the slope is near horizontal, implying statistical independence (per L383). Does that mean, for that part of the range, OI and L4 hSSS accurately reflect hSST, but are independent of hSST?

The accuracy has been defined as the value of conditioned standard deviation for negative singularity exponents only.

o P16L407 - "Hence, the L4 is the most accurate product according to the two defined criteria (extension of the range of singularity correspondence and average conditioned standard deviation)."

- It could be argued that Aq, AqC, and $S G$ appear to have a greater extent where the best fit hSSS:hSST slope is closer to 1 and, therefore, more representative.

Given that the range of uncertainty is larger in the singularity independence domain, it seems that the straight line can be extended for a bit longer and still fit with the range of values

When the modal line (i.e., the line joining all the modes computed for each column of the conditioned histogram)., as a matter of fact, they are not better (not shown).

- Having a table quantifying the best fit slope values for the domain (hSST range) being considered would help the reader 
compare/judge the correspondence of $h S S S$ and $h S S T$ for the various products.

- Also in the table, quantify each product's extent (hSST range) of relevance/correspondence before becoming statistically independent/uncorrelated.

It is not easy to produce such a table, because the transition takes place at an uncertain point difficult of be precisely defined in a mathematical way. We do not have a model for the transition from the singularity correspondence to the singularity independence ranges (and one can be visually mislead is the standard deviation increases in the second part of the curve, as we commented above). As such, we focus on the analysis for $h<0$, since we safely know that for negative singularity exponents we are in the singularity dependence part for all products (we have added a short sentence highlighting this).

o P16L410 - "while the products with the greatest conditioned standard deviations are the two Aquarius products."

- Is the standard deviation assessed/compared over the entire hSST range, or only for the negative hSST range under consideration?

It is computed over all the range, and especially on the singularity independence range. This has been clarified in the text as follows: "while the products with the greatest conditioned standard deviations, especially on the singularity independence range, are the two Aquarius products"

\section{-5. Wavenumber spectra of the singularity exponents}

o P17L420 - "(i.e., there is no privileged point and then it is equivalent to average any function of $s$ on different maps to average it over a large enough map)"

- Incomprehensible - revise

The sentence has been rephrased as: "...given a large enough area the result of the average of any function of $s$ is independent of the 
location of that area"

o P17L422 - C(r), $S(k)=C(k)$

- Define terminology $(r, x, k, C)$

The terminology has been added as follows: "...then the power density spectrum, at the wavenumber $\mathrm{k}, \mathrm{S}(\mathrm{k})$ of the variable $\mathrm{s}$ is equivalent to the Fourier transform of the two-point correlation, $\mathrm{C}(\mathrm{r})$, i.e., $\mathrm{S}(\mathrm{k})=\mathrm{C}(\mathrm{k})$ where $\mathrm{C}(\mathrm{r})$ is given by:

$\mathrm{C}(\mathrm{r})=\langle\mathrm{s}(\mathrm{x}+\mathrm{r}) \mathrm{s}(\mathrm{x})\rangle_{\mathrm{x}}-\langle\mathrm{s}(\mathrm{x})\rangle_{\mathrm{x}}{ }^{2}$

where $r$ is a lag and $\langle.\rangle_{x}$ indicates averaging over all values of $x . "$

o P17L439 - Equation 3

- Define " $\beta$ " and " $h$ "

Now introduced in the text

o P22L567 - Figure 8

- The 95\% confidence interval appears to be plotted for a single point, without discussion or apparent relevance. What is the purpose for plotting the confidence interval for a narrow wavelength range $(\sim 800-1200 \mathrm{~km})$ that is outside the range that is the focus of the paper $(400-800 \mathrm{~km})$.

The $95 \%$ confidence interval in the power spectra plots has been computed for the entire spectrum which is based on the number of individual spectra being averaged.

o P22L574 - “(Figure 5)”

- Is this the correct figure number, or is it $(5,8$, or 9), or is the reference to a figure in Reynolds and Chelton [2010]?

Yes, it is Fig. 5 of Reynolds and Chelton (2010). This has been clarified in the text. 
Agulhas Return Current

o P22L575 - "at the 400-800 km wavelength range, the monthly-averaged PDS slopes of OSTIA can vary from -0.5 to -2 for three different zonal regions."

- Where do the values -0.5 and -2 come from? Visual inspection of Figure 8, for the range of 400-800 km wavelengths, would seem to indicate a relatively consistent slope of about -2.4 , certainly not -

6. 5. Zonal wavenumber spectra for January 2007 (left) and July 2007 (right) for three regions of the World Ocean: the Agulhas Return Current $\left(47^{\circ}-38^{\circ} \mathrm{S}, 45^{\circ}-85^{\circ} \mathrm{E}\right)$, the Kuroshio Extension $\left(30^{\circ}-45^{\circ} \mathrm{N}, 150^{\circ} \mathrm{E}-150^{\circ} \mathrm{W}\right)$, and the western tropical Pacific $\left(5^{\circ} \mathrm{S}-10^{\circ} \mathrm{N}, 150^{\circ} \mathrm{E}-\right.$ $160^{\circ} \mathrm{W}$ ). For each region, wavenumber spectra were computed from daily averaged SST fields in each month along each latitude of grid points within the specified domain. These individual spectra were then ensemble averaged over the latitudes and the 31 days of each month. (top left) The color key for the six SST products is shown.

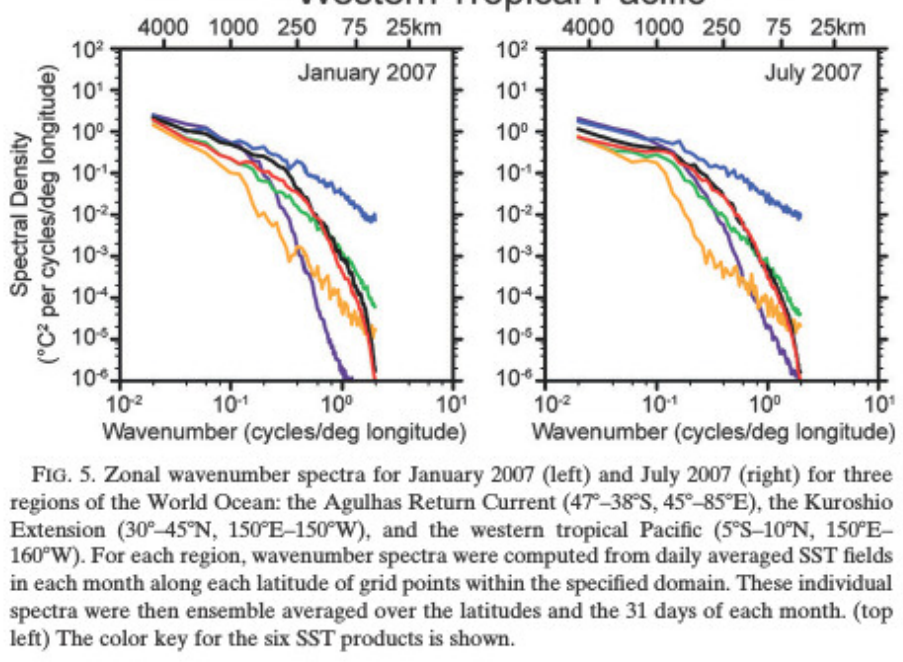


0.5 .

As per previous comment, the sentence refers to Fig. 5 of Reynolds and Chelton (2010).

- Which three different zonal regions? The OSTIA SST slopes for all of the listed regions seem quite consistent, particularly within the designated wavelength range.

See Fig. 5 of Reynolds and Chelton (2010) above. The three regions are the Kuroshio current, the Aghulas return current, and the Western tropical Pacific.

o P22L576 - Figure 10

- Figure 10 is mentioned before Figure 9. Typically, figures are numbered in the order mentioned. However, if the note above about "Figure 5" questioning the figure number is supposed to reference Figure 9 (although that is not consistent within the context of the discussion), then the figure numbering would be correct.

We have moved the related sentence later in the same section when discussing about figure 10 .

o P23L590 - "Contrarily to the OSTIA product (Figure 8), none of the SSS products is able to reproduce the turbulent cascade below $200 \mathrm{~km}$, including the numerical simulation ( $S G)$."

- How are the authors interpreting "reproduce" as applied to the SSS turbulent cascade for wavelengths smaller than $200 \mathrm{~km}$ ?

As stated in the text, the authors use the TO spectral slope of -2.4 as the reference slope at all scales.

- Figure 9.a, b, c, d, g, and h (all products except the WOA 2009 and the two Aquarius products) have turbulent cascades indicated for wavelengths smaller than $200 \mathrm{~km}$. Those SPS slopes do tend to become steeper than -2.4 for the shortest wavelengths, but some of the products/regions remain close to a -2.4 slope.

Indeed, a few products/regions do show a slope close to -2.4. This has been clarified in the text as follows: "...none of the SSS products consistently reproduce the reference turbulent cascade below $200 \mathrm{~km}$ for all regions, including the numerical simulation 
(SG)."

- Figure 9 subplots do not employ consistent labeling of the products, e.g., 9.g (CORA vs SC), 9.h (GLORYS2V3 vs SG), etc.

Corrected.

- Not clear about the purpose of this discussion of short wavelengths, given the paper's explicit focus on assessing performance for wavelength scales between $400 \mathrm{~km}$ and $800 \mathrm{~km}$.

Indeed, the paper focuses on wavelengths between $400 \mathrm{~km}$ and 800 $\mathrm{km}$. But since we assume that the slope of -2.4 holds for all scales, we have briefly discussed as well the inherent limitation of the stateof-the-art SSS products at short scales.

o P24L606 - Figure 10

- For consistence with Figure 7, suggest keeping the PDS plot on the left and the SPS plot on the right. The reader is pre-conditioned for that configuration.

Done, thank you.

o P24L608 - "The smallest range of spectral slopes across the different regions corresponds to the OSTIA SST”,

- How much of the convergence can be attributed to the much higher spatial resolution of SST data with respect to observed phenomena? We have tested the effect of OSTIA SST resolution (from 0.05 to 1degree) on the PDS and found that the large-scale energy level remains the same, while the flattening of the spectra (white noise) occurs at decreasing wavenumber with decreasing SST resolution.

o P24L612 - "The error of these products can easily exceed 0.2 in several regions of the ocean [BEC, 2014; Lagerloef et al., 2015]."

- Establish the relevance of this statement to the discussion of noise. Agree. The last two sentences of the paragraph have been rephrased as follows: "Regarding remotely sensed gridded products, the wide spread of their PDS slopes (Figure 10 left) reveals the impact of noise exceeding 0.2 [BEC, 2014; Lagerloef et al., 2015] in the 
estimated spectra as discussed for Figure 7."

o P24L618 - "reanalysis (SG), the spectral slopes estimated by the standard PDS range from about -0.5 (close to white noise) to -3.5 (lack of intermediate scale variability)"

- What is the basis for this statement? Need to reference Figure 10.

- What is the basis for claiming that a -3.5 slope indicates a lack of intermediate scale variability? "Reduced" variability? At what spectral slope has the slope become "steep" and indicative of an over-smoothed field? A slope of -3.5 is not much different from the reference slope of -2.4.

Agree. We have replace "lack of" by "reduced".

o P24L622 - "In stark contrast, the values of spectral slopes provided by SPS are within the range -2 to -3 , which is physically more plausible"

- Presume that this discussion is in reference to Figure 10. Figure 10 should be referenced.

Agree. The reference of Figure 10 has been added.

- If singularity is approximately independent of the choice of translationally invariant parameter, why are there such slope differences for the SST products TC, TG, T09, and T13, given the finer time/space resolution of SST data? The cascade to smaller scales should be better defined and, at least, consistent with SSS scales.

Good point. We consider TO as the reference product, for which a consistent slope of -2.4 is found in all regions and at all scales. The reason why the other SST products show different SPS slopes is because they are not able to well reproduce the turbulent cascade due to, e.g., interpolation, data gaps, etc. Although the cascade for the same SST and SSS of each product should be the same, this does not happen because the input dataset are different (due to different quality control, data gaps, and data sources). However this raises an interesting point that we plan to further investigate.

The TG, TC, T09, and T13 data/products are not referenced in the References listing. 
The references for SST products have been added.

\section{- 6. Conclusion}

o P26L671 - “detecting artifacts”

- What artifacts? The use of singularity analysis for detecting artifacts was not discussed. Inconsistency perhaps, but is the absence of fine detail considered an artifact?

Rather than the absence of fine detail, it's about how well the singularity fronts (streamlines) are represented or not (e.g., the presence of noise or a geophysical inconsistency breaks up the singularity lines). The word "artifact" has been replaced by "inconsistency"

- Figures

o Figure 1

- title?

The title has been added.

o Figure 2

- Title? Perhaps “Annual SSS mean: 2012”

- Label subplots

- Smallness of subplots renders comparison difficult

- Title color bar, including units as appropriate

The Figure has been modified, adding title and subplot titles.

o Figure 3

- Title? Intra-annual SSS variance: 2012

- Perhaps “Intra-annual SSS variance: 2012”

- Label subplotsSmallness of subplots renders comparison difficult

- Title colo rbar, including units as appropriate 
The Figure has been modified accordingly.

o Figure 4

- Title?

- Recommend plotting the singularity data as a darker color on a white background.

- Title color bar, including units as appropriate

The Figure has been modified accordingly. Note however, that the dark background has been kept (see answer to a previous related comment)

o Figure 5

- Title?

- Label subplotsSmallness of subplots renders comparison difficult

- Title color bar, including units as appropriate

The Figure has been modified accordingly.

o Figure 6 title?

- Title?

- Label subplots

- Title color bar, including units as appropriate

- Smallness of subplots renders comparison difficult

- Labeling too small to read

The Figure has been modified accordingly.

o Figure 7

- Title? 
- The original data curve is not evident; although, it may be completely covered by the 0.10 (magenta) line.

The Figure has been modified accordingly.

o Figure 8 title?

The title has been added to the Figure.

o Figure 9

- P35L902 -Figure 9 caption: Wrong caption, does not match the figure.

- Title?

- Needs color legend

- Labeling too small to read

The Figure has been modified accordingly.

o Figure 10

- Title?

- Axes are not labeled

- Labeling too small to read easily

The Figure has been modified accordingly.

\section{- Grammar and Style}

o General

- “in situ” - no hyphen, italicized per the IEEE style manual Modified.

- “et al." - italicized per the IEEE style manual Modified. 
- When listing subplots in a figure's caption, identify the subplot designation first, then its description:

-Subplot: “a) L3" rather than "L3 (a)"

-Figures 2, 3, 5, 6, and 9

Modified.

- Hyphenate "sea surface salinity”, i.e., "sea-surface salinity”. Likewise for sea-surface temperature Even if we agree that the hyphen between sea and surface is formally the most correct way to write it, we have decided to keep it as two words since this is the usual convention and widely used within the community. Ultimately, the Editor can decide in the proof reading stage about this.. 


\title{
Singularity Power Spectra: A method to assess geophysical consistency of gridded products - Application to sea surface
} salinity remote sensing maps

\author{
Nina Hoareau*, Antonio Turiel, Marcos Portabella, Joaquim Ballabrera-Poy and Jur \\ Vogelzang \\ Institut de Ciències del Mar, CSIC, Barcelona, Spain. \\ *Corresponding author: Nina Hoareau, Departament d'Oceanografia Física i Tecnològica, Institut \\ de Ciències del Mar, CSIC. Passeig Maritim de la Barceloneta, 37-49. Barcelona 08003. \\ Tel.: +34932309500. (nhoareau@icm.csic.es)
}

\begin{abstract}
:
The Soil Moisture and Ocean Salinity (SMOS) and Aquarius satellite missions have produced the first sea surface salinity (SSS) maps from space. The quality of the retrieved SSS must be assessed, not only in terms of its validation against sparse ground truth, but also in terms of its ability to detect and characterize geophysical processes, such as mesoscale features. Such characterization is sometimes elusive due to the presence of noise and processing artifacts that continue to affect state-of-the-art remote sensing SSS maps. A new method, based on singularity analysis, is proposed to improve the assessment of the geophysical characteristics
\end{abstract}


of SSS maps. Singularity analysis can be used to directly assess the spatial consistency of the SSS fields, and to improve the estimation of their spectral slopes in wavenumber spectra through a new method, the so-called Singularity Power Spectra (SPS). To demonstrate its performance and utility, we have applied SPS to different gridded SSS maps, such as SMOS and Aquarius high-level products, the output of a numerical simulation, in situ reanalysis and climatology, as well as to other sea surface temperature products for reference. The singularity analysis and SPS methods reveal that the SMOS Level 4 and the Aquarius Combined Active Passive products are both able to describe the geometry of the existing geophysical structures and provide consistent spectral slopes. This work demonstrates that, beyond the remaining sources of uncertainty in remote sensing SSS products, additional valuable dynamical information on the ocean state can be extracted from these SSS products.

Key words: Sea surface Salinity, Remote sensing, SMOS, Aquarius, geophysical consistence, singularity analysis, singularity power spectra.

\section{Introduction}

Satellite remote sensing measurements have been used in oceanography since the mid-1970s. Thanks to satellite imagery the oceanographic community has been able to better interpret surface structures, such as fronts and eddies, that became apparent in instantaneous views of the ocean. Moreover, satellite altimetry and sea surface temperature (SST) observations highlighted the high percentage of energy accumulated at oceanic mesoscales (tens to hundreds of $\mathrm{km}$ in space and days to weeks in time). Today, thanks to the launch of the Soil Moisture and Ocean Salinity (SMOS) mission (2009) and then the Aquarius mission (2011), more than seven years of satellite-derived sea surface salinity (SSS) data are available. 
Until the advent of SMOS and Aquarius, information about the spatial and temporal variability of salinity had been scarce due to the lack of a comprehensive set of salinity observations. With few exceptions, for example, Taylor and Stephens [1980], Delcroix et al. [2005], or Reverdin et al. [2007], numerical models had been the main source of such information, e.g. Mignot and Frankignoul, [2003], and references therein.

Remote sensing of SSS relies on the sensitivity of the ocean microwave emissivity to changes in sea-water conductivity, which, in turn, depends on salinity. SMOS and Aquarius have been the first SSS missions, with both carrying onboard L-band microwave radiometers. L-band satellite measurements, notably interferometric measurements (SMOS), have proven to be challenging despite the many on-going efforts to improve the quality of retrievals [GonzalezGambau, 2016a, 2016b]. As such, data post-processing is mandatory to recover structured and meaningful geophysical information from remote sensing SSS retrivals.

Direct comparisons against in situ salinity measurements (moored buoys, drifters, and thermosalinographs) have been extensively used to assess the reliability of remotely sensed SSS [Boutin et al., 2012; Reul et al., 2012; Hernandez et al., 2014; Kolodziejczyk et al., 2015a]. This validation approach, however, has some limitations, as in situ data are assumed to be true or perfect. Although the in situ instrument errors are usually considered negligible, some essential differences between remote sensing and in situ data should be considered.

Regarding the direct comparison of remotely sensed retrievals with in situ data, one has to consider data representativeness issues [Stoffelen, 1998; Portabella and Stoffelen, 2009; Vogelzang et al., 2011]. Indeed, in situ data and satellite products likely have different spatial and temporal sampling characteristics, potentially hindering the use of in situ data to verify the spatial and temporal consistency of satellite-derived fields. An in situ observation, e.g., Argo, represents an almost instantaneous and point measurement; whereas, data products, 
e.g., satellite or model output, are given in grids with spatial sizes of 10-100 km, with temporal averages of 1-30 days. Moreover, satellite SSS products measure the top $1 \mathrm{~cm}$ of the ocean surface while in situ SSS measurements are usually obtained few meters below the surface. All of these differences in representativeness should be taken into account during validation. Gridded products may be affected by large-area biases, an especially important consideration in the case of SMOS, where the largest biases include radio-frequency interference (RFI) sources and land-sea Contamination (LSC). These biases may produce mixed or even misleading results. Although some of the techniques proposed to correct LSC in the SMOS data may lead to SSS maps that compare well with the sparse in situ data, these techniques also degrade the geophysical variability signal [Kolodziejzyk et al. 2016; Olmedo et al. 2016]. Hence, direct comparison of in situ and remote sensing data, by itself, does not provide a complete picture of the actual data quality.

Spectral analysis can be adequate for characterizing the geophysical consistency of the retrieved SSS structure, especially for those signals that present clear resonant peaks at specific spatial frequencies corresponding to structures with characteristic sizes. However, except when analyzing areas with persistent geophysical features (e.g., intense mesoscale activity with frequent geostrophic eddies, frontal systems, etc.), these resonant peaks are not present and the wavenumber spectra analysis only provides information about the onset of the turbulent cascade, and how the cascade affects the spatial variability of the field being analyzed. The effect of the cascade on a scalar shows up in the wavenumber spectra, as a power-law scaled by an exponent (spectral slope) that depends on the specific turbulent regime dominating the area. Typical values of the spectral slope (below $300 \mathrm{~km}$ ) for salinity, derived from in situ data are between -1 and -3 [Kolodziejczyk et al., 2015b; Cole et al., 2010; Cole and Rudnick, 2012]. Dispersion in the spectral slope value of a product, depending on the region under study [Reynolds and Chelton, 2010], can be the consequence 
of actual differences in the dynamics of the ocean in the different regions, e.g. large mesoscale SSS variability can be induced by surface atmospheric fluxes, horizontal, and vertical process, but this dispersion also can result from the presence of correlated and uncorrelated noise and processing issues. Considerable uncertainty in the estimation of the spectral slope results, which, in turn, unless the slope largely deviates from the expected range of values, makes it impossible to identify the dominant turbulent regime, considerably hindering the verification of the geophysical consistency of a given parameter.

This work aims to present complementary methods; based on singularity analysis [Turiel et al., 2008a; Turiel et al., 2009], for extracting additional information about the spatial variability from salinity products, including climatology, model output, in situ reanalysis and remote sensing analyses. The quality of a product is assessed according to a new criterion: the ability to describe the actual geometry of flow structures, which are reflected in the shape of features and short-range correlations of the variables. This geometrical assessment can be performed by means of singularity analysis, a powerful data processing technique that permits extracting flow-induced characteristics from any scalar [Turiel et al., 2008a; Turiel et al., 2009]. Additionally, a new method, based on calculating the wavenumber spectra of the singularity exponent fields, i.e., singularity power spectra (SPS), is presented. In contrast with traditional power density spectrum (PDS) analysis, whose small-scale (here defined less than $400 \mathrm{~km}$ ) spectral slopes are very much affected by measurement noise, we show that the SPS technique is able to reveal the small-scale turbulent cascade signature, despite the limitations of the current remote sensing systems, especially in terms of accuracy relative to in situ data, resulting in good representation of the spatial variability SSS.

The outline of this paper is as follows: Section 2 describes of the different data products, regions of interest, and time periods. Section 3 discuss the application of the singularity analysis technique, along with resulting singularity exponent. Section 4 provides an analisis 
of the geophysical consistency of the SSS maps according to the structure of their singularity exponents, using the singularity exponents obtained from the Operational Sea Surface Temperature and Sea Ice Analysis (OSTIA) SST product [Donlon et al., 2012] as reference; Section 5 presents and applies the singularity power spectra (SPS) analysis toward more accurate determining the spectral slopes of the different variables and, finally, Section 6 provides concluding remarks and outlook.

\section{Data and regions}

\subsection{SSS data products}

\subsubsection{SMOS}

The SMOS satellite, launched in November 2009 is part of the European Space Agency's (ESA) Living Planet Program [Mecklenburg et al. 2012], is the first polar-orbiting satellite with a passive microwave sensor operating in the L-Band $(1.4 \mathrm{GHz})$. Using polarimetric interferometry from 69 individual radiometers, SMOS retrieves SSS in a 1200-km-wide field of view, providing global coverage every three days. ESA's Level-2 (L2) swath-based SSS products have a spatial resolution of $30-50 \mathrm{~km}$, but a rather low accuracy of 0.6 to 1.7 [Reul et al. 2012; Boutin et al. 2012]. Note that salinity is defined in the Practical Salinity Scale 1978 [UNESCO 1981], and the salinity values are given without unit. The SMOS SSS products used here correspond to the operational V1.0 generated by the SMOS Barcelona Expert Center (BEC) at 0.25-degree resolution (available at http://cp34-bec.cmima.csic.es). The following three high-level monthly averages are used:

Level-3 binned (SL3), Optimally Interpolated (SOI), and Level-4 (SL4) maps. 
- http://www.cp34-smos.icm.csic.es/:SMOS Level-3 binned (SL3) product: Reprocessed ESA L2 version 550, Ocean Salinity User Data Product (UDP) files (https://earth.esa.int/web/guest/data-access) [SO-TN-IDR-GS-0006 2016], which includes geophysical parameters, a theoretical estimate of parameter accuracy, and various product quality descriptors, are used to generatinge the L3 SL3 product. The information contained in the UDP files is used to filter out uUnreliable values from the L2 SSS data are filtered out using the data quality flags included with each retrieval before bin averaging the data m ointo a 9-day, 0.25-degree grid.

- SMOS Level-3 Optimally-interpolated (SOI) product: The SL3 9-day SSS data are optimally interpolated (OI, Bretherton et al., 1976) to produce maps with having higher spatial and temporal consistency. The SOI is performed usingemploys the monthly World Ocean Atlas (WOA), 2009 [Antonov et al., 2010]) SSS monthly climatology as the a background field and the decorrelation scale described by JordaJorda et al.et al. [2011].

- SMOS Level-4 fused (SL4) product: The data fusion algorithm described by Umbert et al.et al. [2014] is used at BEC to build the SL4 product, in which the SSS SL3 maps and are fused with 9-day OSTIA SST maps [Donlon et al.et al. 2012] are fused, employing. $\mathrm{T}$ the rationale, behind the approach builds upon the notions, and results of the singularity analysis described in Turiel et al.et al. [2008b]. The resulting SL4 product is a 9-day mean at 0.25 -degree grid resolution.

Detailed descriptions of these products are given in [BEC 2013], and their corresponding quality reports are given in BEC [2014].

\subsubsection{Aquarius}


The Aquarius instrument, onboard the Aquarius/SAC-D satellite, operated from its launch in June 2011 until June 2015 as a collaborative effort between the National Aeronautics and Space Agency (NASA) and the Argentine Space Agency Comisión Nacional de Actividades Espaciales (CONAE). The instrument consisted of three real-aperture radiometers in a push broom configuration at 29-, 38-, and 46-degrees incidence angles relative to the shadow side of the $6 \mathrm{am} / 6 \mathrm{pm}$ sun-synchronous orbit. The beam footprints were $76 \mathrm{~km}$ (along-track) x 94 km (cross-track), $84 \mathrm{~km}$ x $120 \mathrm{~km}$ and $96 \mathrm{~km}$ x $156 \mathrm{~km}$, respectively, yielding a combined swath cross-track width of $370 \mathrm{~km}$. As SMOS, the radiometers measured the brightness temperature at $1.4 \mathrm{GHz}$ in the L-Band. The platform also carried a scatterometer, operating at 1.26 GHz, to measure the ocean backscatter in each footprint, for correcting sea surface roughness effects, thereby improving salinity retrievals. This data is available at ftp://podaacftp.jpl.nasa.gov/allData/aquarius/docs/v4/AQ-014-PS-

0016_AquariusSalinityDataValidationAnalysis_DatasetVersion4.0.pdf.

Two Aquarius SSS monthly products, with a one-degree-grid resolution are used:

- Aquarius v4 (Aq) product: The Level 3 product is created with version 4 of the Aquarius/SCA-D operational processor (https://podaac.jpl.nasa.gov/announcements/2015-0717 OFFICIAL NASA AQUARIUS/SAC-D VERSION 4.0).

- http://podaac-opendap.jpl.nasa.gov/opendap/allData/aquarius/L3/mapped/V3) or Aquarius v3 (Aq) product, and the Aquarius CAP (AqC) product [Yueh et al., 2014]:: The CAP algorithm [Yueh et al., 2014] simultaneously retrieves salinity, wind speed, and wind direction by minimizing the sum of squared differences between model values and observations.

\subsubsection{GLORYS2V3 Reanalysis}


The Mercator Ocean Global Ocean Physics Reanalysis GLORYS2V3 1993-2013 (SG for SSS and TG for SST data), available from the Copernicus Marine Environmental Monitoring Service (CMEMS, http://marine.copernicus.eu), uses the Nucleus for European Modelling of the Ocean [NEMO, Madec et al., 2012] version 3.1 ocean model in the ORCA025_LIM configuration, [Parent et al., 2013]. The model has $1 / 4^{\text {th }}$-degree horizontal resolution and 75 levels, with a partial step at the bottom. The ERA-interim forcing fields include a large-scale correction for downward (shortwave and longwave) radiative and rainfall fluxes. This simulation uses the LIM2 thermodynamic ice model with an elastic-viscous-plastic rheology formulation. The data assimilation technique is a multi-data and multivariate reduced order Kalman filter based on the Singular Extended Evolutive Kalman (SEEK) filter. A bias correction scheme for temperature and salinity is also included. Increments are applied using an Increment Analysis Update. The assimilated observations are delayed-time along-track satellite sea level anomaly, sea ice concentration, SST, and in situ profiles of temperature and salinity from the Coriolis Ocean Dataset for Reanalysis version 3.3 (CORA3.3) database [Cabanes et al., 2013]. No restoring terms for temperature or salinity are used in this configuration.

The original fields of the ORCA025_LIM irregular grid (nominal $0.25^{\circ}$ grid at the equator) are interpolated onto a regular $0.25^{\circ}$ global grid using a squared-distance weighted interpolation scheme in order to simplify calculating the singularity power spectra (SPS).

\subsubsection{COriolis Ocean Dataset for Reanalysis (CORA)}

The monthly CORA (SC for SSS and TC for SST) fields, at 0.5-degree spatial resolution are generated by the objective analysis of the global, delayed-mode in situ observations available from the Copernicus in situ Thematic Assembly Center (TAC) (global component) operated 
by the Coriolis data center and available from CMEMS. This system is based on a statistical estimation method (objective analysis) using the ISAS software [Gaillard et al. 2016].

\subsubsection{World Ocean Atlas climatology}

The three-dimensional monthly salinity and temperature climatologies from the National Oceanic and Atmospheric Administration's (NOAA) World Ocean Atlas 2009 [S09 for SSS, Antonov et al., 2010; T09 for SST, Locarnini et al., 2009] and 2013 [S13 for SSS, Zweng et al., 2013; T13 for SST, Locarnini et al,. 2013], created by optimal interpolation of all the available historical sets of in situ data, are used. The horizontal grid resolution is one-degree for S09, T09 and 0.25-degree for S13 and T13..

\subsection{SST data products: OSTIA Sea surface Temperature}

In addition to the salinity products described above, we also use the daily SST maps of the TO generated by the Group for High Resolution SST (GHRSST). The OSTIA SST (TO) maps are produced daily at $1 / 20^{\text {th }}$-degree resolution by applying an adapted version of the optimal interpolation algorithm to combine satellite and in situ observations.

\subsection{Regions and time period}

The spatial variability of the SSS products is assessed through the analysis of the meridional and zonal variability within the six regions shown in Figure 1. Two regions have a meridional extent: the North Atlantic Ocean (NATL: $0^{\circ} \mathrm{N}-64^{\circ} \mathrm{N}, 27^{\circ} \mathrm{W}-33^{\circ} \mathrm{W}$ ) and the South Pacific Ocean (SPAC: $50^{\circ} \mathrm{S}-14^{\circ} \mathrm{N}, 128^{\circ} \mathrm{W}-122^{\circ} \mathrm{W}$ ). The other four regions are zonal: the Pacific Inter-Tropical Convergence Zone (ITCZ: $4^{\circ} \mathrm{N}-10^{\circ} \mathrm{N}, 160^{\circ} \mathrm{W}-96^{\circ} \mathrm{W}$ ) characterized by the presence of the eastern fresh pool, the region of North Atlantic Subtropical Gyre characterized by the local maximum of salinity where the Salinity Processes in the Upper- 
ocean Regional Study (SPURS) campaigns took place in 2012 and 2013 (SPURS: $22^{\circ} \mathrm{N}$ $28^{\circ} \mathrm{N}, 60^{\circ} \mathrm{W}-28^{\circ} \mathrm{W}$ ), the Agulhas Return Current (ARC: $45^{\circ} \mathrm{S}-39^{\circ} \mathrm{S}, 30^{\circ} \mathrm{E}-94^{\circ} \mathrm{E}$ ), and the South Tropical Pacific Ocean (STP: $33^{\circ} \mathrm{S}-27^{\circ} \mathrm{S}, 170^{\circ} \mathrm{W}-106^{\circ} \mathrm{W}$ ).

Despite the meridional changes in the first baroclinic Rossby radius of deformation across the SPAC and NATL box regions, the SPS analysis is performed in the meridional direction because we are interested in knowing whether the turbulent cascade verifies differently along the meridional and the zonal directions. Besides, due to the the meridional boxes being similarly oriented to SMOS and Aquarius ground tracks, SSS observations within those boxes are more synoptic, so fewer artifacts are anticipated. In the other four regions, the SPS analysis is carried out in the zonal direction, i.e., with approximately constant Rossby deformation radii.

The time period selected for this analysis is the year 2012 when all of the SSS and SST products are available and because a significant number of ground-based RFI sources identified during the first year of both missions had already been eliminated.

\subsection{Mean and variance of SSS products for 2012}

Figures 2 and 3 show, respectively, the annual SSS mean and intra-annual variance of the nine monthly products described in Section 2.1. The main structures of the annual mean are similar among all these products; although, some significant differences exist between them, including the spatial extent (near the coast and at high latitudes) of salinity maxima, the salinity range, and the presence of small scale noise in remote sensing products. Notice that SMOS products (Figures $2 \mathrm{a}, 2 \mathrm{~b}$, and $2 \mathrm{c}$ ) fail to locate the subtropical salinity maxima as close to the coast as the other products do, especially in the case of the South Atlantic 
subtropical gyre. This artifact is due to the high sensitivity of the synthetic aperture image reconstruction to land-sea contamination. Important differences exist, in both spatial structure and amplitude, between the variance maps (Figure 3). The largest SSS annual variances are associated with the Amazon and Congo runoffs, the ITCZ eastern fresh pool, high latitudes, monsoons, the gulf of Tehuantepec, and the Gulf Stream. In the western part of the Equatorial Pacific Ocean (see highlighted box in Fig. 3h), the signal of La Niña (from November 2011 through April 2012) is captured by both SMOS (Figures 3a, 3b and 3c) and Aquarius (Figures 3f and 3i) as well as by the numerical simulation (Figure $3 \mathrm{~h}$ ) and the in situ reanalysis (Figure $3 \mathrm{~g}$ ). The variance maps show similar variability levels in the Aquarius (Figures $3 \mathrm{f}$ and $3 \mathrm{i}$ ) and SMOS OI (Figure 3b), with only a few regional differences being seen, e.g., the Amazon plume. These differences are mostly associated to the lack of SL3 data (Figures 2a and 3a) in these regions. As already indicated by Umbert et al. (2014), the fusion algorithm results in a smaller dynamic range of the fused field, e.g. SL4 (Figures 3c). In comparison with SG and in situ products, the remote sensing products show extended regions of higher variability in the southern ocean. In particular, south of $60^{\circ} \mathrm{S}$, the SL3 product displays a variance that is substantially larger than that of the other remote sensing products. This variance is artificial and is related to the low sensitivity of the L-Band brightness temperature to salinity changes at low SST. Moreover, SMOS SSS retrievals are very much constrained by European Centre for Medium-Range Weather Forecasts (ECMWF) winds [Montuori et al., 2013], which are particularly inaccurate for high wind regions, such as the Southern Ocean. High-level SMOS products, as SOI and SL4, depict a significant reduction of such noise.

\section{Singularity analysis}


The spatial coherence of a scalar, e.g. as temperature or salinity, advected by a turbulent flow can be assessed through singularity analysis [Turiel et al., 2008b]. Singularity analysis refers to any technique capable of assigning a singularity exponent to each point in a map of a given variable. The singularity exponent at a given point is a measure of the regularity or irregularity of the variable around that point. The singularity exponent generalizes the concept of the number of admissible derivatives at any given point, allowing for non-integer derivatives. The oceanographic interest in singularity exponents arises because they are a characteristic property of the flow [Turiel et al., 2005; Turiel et al., 2008a; Turiel et al., 2009] and not specific to the chosen scalar [Nieves et al., 2007]. In this sense, singularity exponents are related to the advection term, representing the part of the signal that all ocean surface scalar fields share. It must be noted that the singularity structure does not carry information about the amplitude of the changes of the variables for the depicted geophysical structures, only about their geophysical consistency (i.e., whether they represent similar currents, fronts, eddies, etc.). Thus, we can assess the quality of any ocean scalar by simply analyzing whether their singularity exponents (and related statistics) correspond to those of a reference variable. The presence of long-range correlations, either of geophysical origin or caused by artifacts, does not modify the singularity exponents [Turiel et al., 2008a].

The singularity exponents of the different salinity products have been calculated using the same dedicated software presented in Turiel et al. [2008a] (The Barcelona Expert Center offers a free web service that allows users to obtain the singularity exponents associated with any scalar field contained in a NetCDF file. This service can be accessed at http://cp34bec.cmima.csic.es/new-service-available-singularity-analysis/). The resemblance between the structures in the singularity maps of SST and SSS has already been shown in Umbert et al. [2014] and Hoareau et al. [2014]. Figure 4 represents the map of singularity exponents derived from the TO map of January 2012. Due to the high quality of the OSTIA SST fields, 
the associated singularity maps will serve as the reference for comparison with the other SSS products in this study. Visual inspection of the singularity map reveals its geophysical coherence, i.e., the singularity fronts (bright white streamlines) are clearly associated with large- and small-scale features of the global circulation, the Gulf Stream and Kuroshio, the multiple fronts of the Antarctic Circumpolar Current, the Tropical Instability Waves in both the Pacific and the Atlantic, etc. In fact, as shown in Figure 9 of [Turiel et al., 2009], singularity fronts derived from OSTIA SST align very well with the streamlines of the flow, so we consider OSTIA SST SE a good reference at the scales considered in this paper. As expected, individual mesoscale eddies cannot be observed in monthly products. Figure 5 shows the singularity exponent maps derived from the nine monthly SSS products in January 2012. In contrast with the SST singularity map (Figure 4), the SL3 singularity map (Figure 5a) appears completely unstructured: only a few strong signals, such as the Orinoco and Amazon plumes, can be recognized. Since the SL3 product is based on a monthly average of swath-based L2 averaged (binned) data, Figure 5a is mainly driven by the low signal-to-noise ratio of the SMOS L2 data [BEC, 2014]. Only after applying effective noise reduction techniques to the SMOS data, such as optimal interpolation (Figure 5b) or data fusion (Figure 5c), the singularity fronts or structures (streamlines) reflect those of the SST map (Figure 4).

The singularity maps derived from S13 and S09 SSS climatologies (Figures 5d and 5e) have different features. The S09 singularity map only shows large-scale patterns, while S13 better represents intermediate and small scales and, visually, is more realistic.

For the Aquarius products, which have the same grid resolution as S09, the Aq (Figure 5f) and AqC (Figure 5i) singularity maps visually seem able to resolve some intermediate scale structures, in addition to those of larger scales. 
The SC singularity map is as smooth as S09; thus, it is not able to represent the intermediate and small scales. Finally, the SG singularity map (Figure 5h) reproduces all the streamlines, at all scales, with a similar quality to that of the TO singularity map (Figure 4).

According to the qualitative assessment of the singularity maps presented in this section, the singularity exponents display that some SSS products lack information on the small and even intermediate scales. Singularity exponents are estimated by calculating the slope of a log-log regression of wavelet projections versus wavelet scale [Turiel et al., 2008b]. The wavelet projection of any map regularized by a convolution kernel will be similar as having projected the signal on the wavelet basis at larger scales, adding the characteristic scale of the convolution kernel to the wavelet scale. Therefore, the resulting map of singularity exponents will be of lower resolution (evidenced by thicker singularity lines), and quite naturally would show up as a premature damping of the power spectra at high wavenumbers. This qualitative assessment provides some intuition about what is observed while the agreement between the SSS singularity maps and the reference map is quantified in the Section 4.

\section{Geophysical consistency between SST and SSS}

As already discussed, the singularity exponents obtained from different ocean variables should be the same. In the following, we present a method to evaluate and quantify geophysical consistency between the different SSS products and the TO product in terms of their singularity structure.

To quantify the correspondence between derived singularity exponents, the histogram of SSS singularity exponents $\left(\mathrm{h}_{\mathrm{SSS}}\right)$, conditioned by each SST singularity exponent value $\left(\mathrm{h}_{\mathrm{SST}}\right)$, is calculated, as in Umbert et al. [2014]. This conditioned histogram evidences any possible 
correlation between both variables. According to previous studies [Umbert et al., 2014; Olmedo et al. 2016], when the maximum probability line (modal line, defined by the maximum probability of $h_{\text {SSS }}$ for each value of $h_{\text {SST }}$ ) follows a straight line of slope equal to 1 for the majority of the range of $\mathrm{h}_{\mathrm{SST}}$, then the singularity exponents of the SSS maps are in good correspondence with those of the reference. However, as the singularity estimation error increases with increasing $h_{S S T}$ values [Turiel et al., 2006], it is also expected that this line eventually becomes horizontal beyond a given point, indicating that the SSS and SST singularity exponents are statistically independent for values of $\mathrm{h}_{\mathrm{SST}}$ larger than a certain threshold. Thus, a high-quality SSS product will present a wide range of singularity correspondence with the reference SST singularity exponents (a straight line of slope 1), and a small range of singularity independence (horizontal line). Conversely, a low-quality SSS product will have a reduced range of singularity correspondence with the reference. Note that when two variables are completely independent, the conditional histogram will present a full range of singularity independence. At the same time the conditioned dispersion will be the largest, since any kind of dependence between the variables would tend to reduce the dispersion. Hence, besides analyzing the extent of the singularity correspondence (i.e., range of values of $\mathrm{h}_{\mathrm{SST}}$ for which the maximum probability is located along the diagonal), the conditioned dispersion (i.e., for each value of $\mathrm{h}_{\mathrm{SST}}$, the degree of dispersion of the associated column, as in Umbert et al, 2014) also must be analyzed.

Figure 6 shows the joint histogram of the singularity exponents, conditioned by the singularity exponents of TO, associate with each SSS product. We observe that the singularity exponents are independent in most of the $\mathrm{h}_{\mathrm{SST}}$ range, indicating that the error level is rather large for all SSS products. The analysis will be limited to the range of negative singularity exponents of SST, which is where singularity correspondence typically is evidenced; this range is taken as $\mathrm{h}<0$. Note, though, that some isolated points are vertically 


\section{Wavenumber spectra of the singularity exponents}

\subsection{Singularity power spectra (SPS) method}

If a variable $s$ is translational invariant (i.e., given a large enough area the result of the average of any function of $s$ is independent of the location of that area.), then the power density spectrum, at the wavenumber $k, S(k)$ of the variable $s$ is equivalent to the Fourier transform of the two-point correlation $C(r)$, i.e., $S(k)=C(k)$, where $C(r)$ is given by

$C(r)=\langle s(x+r) s(x)\rangle_{x}-\langle s(x)\rangle_{x}^{2}$

where $\mathrm{r}$ is a lag and $\langle.\rangle_{x}$ indicates averaging over all values of $x$. Using the fact that the spectral slope of any variable is the same for any bi-Lipschitz transformation [Rudin, 1987], 
we use some functional relations to define a new way of deriving the spectral slope of noisy. A bi-Lipschitz transformation on a real domain $U$ is a real function $F: U \rightarrow R$ such that there is a constant $A>0$, which verifies that, for any $x, y \in U$, the following relation holds:

$A^{-1}|x-y| \leq \vee F(x)-F(y) \vee \leq A|x-y|$

Hence, if the two-point correlation of a variable s presents a power-law scaling $C_{s}(r) \propto r^{\gamma}$, and if $F$ is bi-Lispchitz, then the two-point correlation of $F(s)$ scales the same way, i.e. $C_{F(s)}(r) \propto r^{\gamma}$, automatically implying that the power spectra of $s$ and $F(s)$ have the same spectral slope.

Accordingly, we can use the singularity exponents of the variable s to improve the estimation of the slope of the wavenumber spectra. According to [Turiel et al., 2008b], the gradient of any scalar $s$, as estimated at a given finite scale $r_{0}$, behaves like

$\nabla_{r_{0}} s(x) \approx \mathrm{V} s\left(x+r_{0}\right)-s(x) \mathrm{V} \approx \beta(x) r_{0}^{h(x)}$

where $\beta$ stands for the amplitude of the gradient, that does not depend on the scale $r_{0}$, and $\mathrm{h}(\mathrm{x})$ is the singularity exponent of the signal at that given point. By taking logarithms to both sides of the equation we have:

$\log \left(\left|\nabla_{r_{0}} s(x)\right|\right) \approx \log \beta(x)+h(x) \log r_{0}$

The logarithm is a bi-Lipschitz function on the real positive numbers. If the resolution scale is small enough, compared to the integer scale of the map, then the absolute value of the logarithm becomes large and then the first term on the right hand side of equation (4) is negligible in comparison to the second term. Therefore, the spectral slope of the gradient of $s$ is equal to the spectral slope of the singularity exponents $h$. 
On the other hand, the PDS of the gradient can be related to the PDS of the signal by means of a trivial $k^{2}$ factor, namely

$$
\mathrm{S}_{\nabla \mathrm{s}}(\mathrm{k})=|\widehat{\nabla \mathrm{s}}|^{2}(\mathrm{k})=\mathrm{k}^{2}|\hat{\mathrm{s}}(\mathrm{k})|^{2}=\mathrm{k}^{2} \mathrm{~S}_{\mathrm{s}}(\mathrm{k})
$$

From (5), it can be concluded that the spectral slope associated with the map of singularity exponents can be related to the spectral slope of the signal $\alpha_{s}$ by means of the following relation:

$\alpha_{s}=\alpha_{h}-2$

Using (1)-(6), the Singularity Power Spectrum (SPS) can be defined as

$P(k) \equiv k^{-2} S_{h}(k)$

When defined as in (7), the SPS should have the same spectral slopes as the original PDS in (5), but it will be less affected by noise (using a good numerical implementation of singularity analysis, as in Pont et al, 2013) and local effects, which are isolated in factor $\beta(x)$ and, removed in the computation of $S_{h}(k)$ when, neglected if the resolution is fine enough, i.e., when $r_{0}$ is small as compared to the size of map.

Wavenumber power spectra (both PDS and SPS) have been calculated for the different ocean regions shown in Figure 1. The spectrum calculation software used in this study is based on the Wind Spectra Calculation code (WSC) developed by the Royal Netherlands Meteorological Institute (KNMI) to compute sea surface wind vector spectra from scatterometer data [Vogelzang et al., 2011]. This study adapts the code for computing spectra of a scalar variable (i.e., temperature, salinity, exponents of singular analysis). Given a sample of $\mathrm{N}$ observations separated at regular distances, the spectrum is calculated using the 
sampling method as the periodogram of the sample. The spectrum is given by the square of the absolute value of the Fast Fourier Transform (FFT) at each frequency component [Vogelzang 2013].

To avoid the aliasing due to the existence of unresolved large scales [Errico, 1985], a detrending technique is required. Otherwise, salinity values of the first and the last point of the sample may significantly differ, leading to a $\mathrm{k}^{-2}$ contribution at the tail of the spectrum (short scales) as discussed Vogelzang [2013]. The linear detrending used here is the linear transformation [Frehlich and Sharman, 2008] that consists of subtracting the linear function passing through the two end points.

The power spectra shown here are given as a function of wavenumber $\left(\right.$ degree $\left.^{-1}\right)$ values. The conrresponding wavelength values $(\mathrm{km})$ are also provided at the top axis of each plot. Note that, although it is qualitatively true that long (short) wavelengths correspond to large (small) spatial scales, Vogelzang et al. [2015] showed that there is no one-to-one relationship between the wavelengths obtained from a power spectrum and the actual spatial scales resolved by the observing system. Consequently, and to avoid misleading interpretations, we will provide interpretation of the power spectra in terms of wavenumber $(\mathrm{k})$ or wavelength $\left(\mathrm{k}^{-}\right.$ $\left.{ }^{1}\right)$ values, mentioning spatial scales only in a more qualitative sense (i.e., large, intermediate, and small scales).

\subsection{Sample pre-processing}

Individual PDS and SPS are computed from each one-dimensional track extracted from the geophysical parameter (SSS) map and the singularity exponent (SST) map, respectively, with the same pre-processing applied to both SSS and SST maps. Each track is one gridpoint wide, 
so the spatial width depends on the resolution of the product, and has a length equal to the corresponding zonal/meridional dimension of each region of interest. When gaps exist, as it may sporadically happen in remote sensing products, a linear interpolation is applied within the affected track. The impact of these sporadic gaps in the resulting power spectra has been investigated and found to be small. All regions, except SPURS (32-degree long), span 64 degrees to allow proper spectrum calculation, given the variety of the spatial scales characteristic of the ocean mesoscale in the different regions depicted in the map of the baroclinic Rossby radii of deformation (Figure 1).

The grid spacing of Aquarius and S09 is one degree while that of SC is 0.5 degrees, and that of SMOS, SG and S13 is 0.25 degrees. Since the length of the 1D tracks is fixed, different grid sizes translate to different numbers of samples: $\mathrm{N}=64$ for the one-degree grids, $\mathrm{N}=128$ for the half-degree grids and $\mathrm{N}=256$ for the quarter-degree grids.

Each individual spectrum (obtained from a single 1D track) is noisy, as the expected value of the error in a spectrum estimate equals the value of that estimate itself [Press et al., 1989; Percival and Walden, 1993]. The mean spectrum for a region is obtained by averaging the individual spectra for all 2012 to obtain a reduced-noise annual mean. Following Emery and Thomson [2001], the 95\% confidence interval corresponds to the 168 degree of freedom (corresponding with the number of tracks averaged for each annual mean spectrum) of the coarse resolution products. For the half-degree and the quarter-degree resolution products, the degree of freedom are 312 and 600, respectively.

The spectral range has been split into three different wavelength intervals: long wavelengths (wavenumbers less than $\left.(1000 \mathrm{~km})^{-1}\right)$, intermediate wavelengths, and short wavelengths (wavenumber greater than $\left.(400 \mathrm{~km})^{-1}\right)$. We focused the power spectra analysis on the intermediate range (wavenumbers ranging from $(400 \mathrm{~km})^{-1}$ to $\left.(800 \mathrm{~km})^{-1}\right)$, where both larger- 
scale aliasing and small-scale noise are not expected to significantly perturb the spectral signal. This intermediate wavenumber range was defined for comparing the different products. Note that power spectra slope at this range have been calculated using the standard linear regression formulation. Also note that, following Equation 4, a factor of 2 has been subtracted from the SPS slopes reported in the next section to permit comparing them to the PDS slopes.

\subsection{Results}

\subsubsection{PDS and SPS sensitivity to noise}

To assess the relative performance of the PDS and SPS analyses, we applied both methods onto the same SSS map of the STP region, simulating different white noise level for the same SG snapshot (June 2013). In total, ten new instantiations were created from the original SSS maps by adding white noise with standard deviations ranging from 0.10 to 1.00 in steps of 0.10 .

Figure 7 depicts the PDS (left) and the SPS (right) for the STP region for the six selected noised SSS maps, plus the original PDS and SPS (black line). All SPS spectra have the same energy level. This is due to the fact that singularity analysis is able to filter out uncorrelated noise. However, in the case of the PDS, as the level of noise increases, the energy of the spectra increases and the spectral slope flattens at a lower wavenumber (as it should by definition).

All spectra from SPS are very close to each other and have a spectral slope of about -2.4 , with slight differences on the shape of the spectra depending on the amplitude of noise. These results show that the SPS produces good results in capturing the geophysical signal, even if it 


\subsubsection{Application of SPS to remote sensing data.}

Figure 8 provides the TO SPS of the selected regions for 2012. The derived spectral slopes are between -2 and -2.5 (the averaged slope is about -2.4 when considering all regions and all scales). The differences in amplitude (which show up as vertical shift) seem to depend on the concentration of singularity fronts in each region. These SPS suggest that, when spectra are averaged over a year, the shape of the turbulent cascade should be similar, even for regions with different Rossby radii. Regional differences mainly translate as different energy levels (i.e., SST spatial variability). In contrast, when considering monthly-averaged PDS, the slope of OSTIA can vary from -0.5 to -2 for different zonal regions as shown in Figure 5 of Reynolds and Chelton [2010].

Moreover, as shown in Figure 7, the presence of white noise can significantly distort the PDS slope even for wavelengths greater than $400 \mathrm{~km}$, which in turn can contribute to widening the spread of the PDS slope for a region. We, therefore, conclude that the small spread of the SPS slopes is physically plausible: consequently the TO SPS averaged slope $(-2.4)$ will serve as the reference hereafter.

Figure 9 depicts the SPS of the nine SSS products in this study. Similar SPS spectral slope to that of the TO $(-2.4)$ are seen for all regions and scales of the following: SOI and SL4 (Figures 9b and 9c), Aquarius products (Figures 9f and 9i), SG (Figure 9h) and S13 (Figure 9d). At short scales (wavelength less than $400 \mathrm{~km}$ ), there are subtle differences among the 
products. In particular, note that the Aquarius products maintain the reference slope up to its shortest scales $(200 \mathrm{~km})$ : whereas, the other products present a slightly steeper spectrum at wavelengths less than $300 \mathrm{~km}$. Comparing the SPS slopes of the products below $200 \mathrm{~km}$ with those of the TO product (Figure 8), none of the SSS products consistently reproduce the reference turbulent cascade below $200 \mathrm{~km}$ for all regions, including the numerical simulation (SG).

The remaining products (L3, SC, and S09) present steeper spectra for certain regions and scales. The SC slope depends on the region, being close to -2.4 at all scales in ARC, SPAC and STP, but becoming steeper for the wavelength shorter than $500 \mathrm{~km}$ in the SPURS, ITCZ and NATL regions. Note that the SL3 spectrum in the STP region is close to -2.4 at all scales, which can be explained by the fact that the STP region is where the SMOS target calibration is applied [Gourrion et al., 2012; 2013]. Both optimal interpolation and data fusion algorithms are able to maintain the true relationship between scales when present in the input data (note that the SL3 product is used as input to both the SOI and SL4products).

Beyong STP region, substantial errors in the SL3 product distort the SPS slope, indicating that retrieval errors are not uncorrelated (see discussion for Figure 7). However, as seen in Figure 9a-c, it is evident that the SOI and SL4methodologies effectively filter both correlated and uncorrelated noise present in the input SL3 product, thus producing to SPS slopes close to -2.4 .

Finally, Figure 10 compares the values of the PDS (left) and SPS (right) spectral slopes intermediate wavelength range derived from each of the products. The TO has the smallest range of spectral slopes across the different regions. However, the range of PDS slopes of TO is wider than that of the SPS, further confirming that different spatial variability regions should result in different spectral energy levels rather than in different slopes, and also that 
the PDS is more sensitive to the presence of noise (see Figure 8). Using the SPS slopes of TO as the reference, it is concluded that the wider spread of slopes present in the other products does not have a geophysical origin. Regarding remotely sensed gridded products, the wide spread of their PDS slopes (Figure 10 left) reveals the impact of noise exceeding 0.2 [BEC, 2014; Lagerloef et al., 2015] in the estimated spectra as discussed for Figure 7.

In the case of the salinity field from the SG, the spectral slopes estimated using the standard PDS methodology range from about -0.5 (close to white noise) to -3.5 (reduce intermediate scale variability). At the intermediate wavelength range, no physical processes can explain slopes close to zero (very decorrelated, hence a very noisy signal) or those that are very steep (oversmoothed field, indicating strongly correlated values). In stark contrast, the values of spectral slopes provided by SPS are within the range -2 to -3 (see Figure 10), which are physically more plausible not only because of the expected consistency among the different regions but also because the results are closer to those reported by previous authors using in situ thermosalinograph (TSG) data, albeit for wavelengths below $300 \mathrm{~km}$ (Cole et al., 2010; Cole and Rudnick, 2012).

From Figure 10, it can be concluded that L4, AqC, and SG present the smallest dispersion in slope values between -2 and -3 across the selected regions, indicating that these products, and notably the L4, better resolve the finer (greater than $200 \mathrm{~km}$ ) structures of SSS fields.

\section{Conclusion}

In this paper, the geophysical consistency of different SSS products has been investigated with the help of singularity analysis and singularity spectral analysis. Different salinity products have been compared to assess their ability to describe geophysical structures and 
their correspondence with the expected scaling associated to the turbulent cascade. The nine SSS products analyzed are: SMOS L3, SMOS OI, SMOS L4, Aquarius Level-3 and CAP (version 4), GLORYS2V3, the CORA reanalysis and the WOA 2013 and the WOA 2009 climatologies.

Visual inspection of the derived singularity maps reveals that some SSS products lack information about the small and even intermediate scales. For instance, the unstructured geometry of SL3 singularities reflects the low signal-to-noise ratio of the SMOS data. By applying effective noise filters to the SMOS data (e.g., higher-level SMOS SOI and SL4products), the derived singularity structure, which is known to depict streamlines, is similar to that derived from high-quality SST maps, e.g., OSTIA SST (TO). In the case of Aquarius (Aq and $\mathrm{AqC}$ ) singularity maps, the large-scale structures are present, but smallscale structures are not discernible. For the SSS of WOA 2009 (S09) singularity map, while depicting a few large-scale patterns, no intermediate- and small-scale geophysical patterns can be seen. In contrast, the SSS of WOA 2013 (S13) and CORA reanalysis (SC) singularity maps show that, with the finest grid resolution, intermediate-scale geophysical structures can be seen. Finally, the SSS of GLORYS2V3 (SG) singularity map well reproduces the primary streamlines, as well as the more detailed ocean structures seen in the OSTIA SST singularity map.

The histogram of singularities of each SSS product, conditioned by the OSTIA SST singularity exponents, was calculated to assess the quality of the singularity exponents. Joint SSS-SST histograms reveal that the error level is rather large for all of the SSS products, as evidenced by the relatively large extent of singularity independence. When focusing on the range of the SST negative singularity exponents, typically where the singularity correspondence occurs, SL4has the best scores in terms of the defined singularity correspondence and conditioned standard deviation criteria. 
An alternative method to the standard power spectra density (PDS), the singularity power spectra (SPS) method, is presented and used to better discriminate the spectral signature of the different SSS products. The results show that, at intermediate scales, the L4, AqC, and SG products have the average and spread of SPS spectral slopes in best agreement with those of the reference OSTIA SST (TO).

With the help of the new singularity spectral analysis, it can be concluded that: i) the SPS of SL3 shows excessive variance and slopes that are the most incompatible with the rest of the products; ii) the processing updates used to generate S13 over S09 are justified from the point of view of the spatial spectra; iii) the large scale variability of the rest of the products are equivalent (scales larger than $800 \mathrm{~km}$ ); iv) below $800 \mathrm{~km}$, the slope of the CORA salinity product (SC) becomes too steep in some regions; v) below $400 \mathrm{~km}$, only the satellite and the model output surface salinities share a slope similar to that of TO; and finally, vi) for shorter scales (below $200 \mathrm{~km}$ ), and despite the fact that no product has the same slope as the reference temperature field, the SL4 remains the product whose slope is the closest to that reference.

As a summary, it can be concluded that the SL4, AqC, and SG products are the most geophysically consistent (in terms of both the singular analysis and the SPS) out of the nine products investigated here.

From a methodological point of view, singularity analysis provides a very effective tool for detecting artifacts in the different remote sensing products and, as such, it provides useful feedback for further product improvements. Future work will, therefore, focus on improving the SMOS products, notably the SOI and the SL4products, using spectra and singularity analyses (among others) as tools for product verification metrics. 


\section{Acknowledgements}

This study was funded by the Spanish R+D Plan under project Promises (ESP2015-67549C3-2) and previous grants. The authors would like to thank SMOS BEC team and in particular, E. Olmedo, J. Martínez, and E. García-Ladona for their interesting comments/discussions and help with the data processing. We greatly thank the reviewers and the editor for improving very much the quality of the manuscript. The Aquarius and SMOS products used in this study can be found at http://podaac-opendap.jpl.nasa.gov and http://cp34bec.cmima.csic.es. CORA and GLORYS reanalysis, and OSTIA SST fields were downloaded from the Copernicus Marine Environment Monitoring Service (CMEMS, http://marine.copernicus.eu). 


\section{References:}

J.I. Antonov, D. Seidov, T. P. Boyer, R. A. Locarnini, A. V. Mishonov, H. E. Garcia, O. K. Baranova, M. M. Zweng, and D. R. Johnson, World Ocean Atlas 2009, Volume 2: Salinity. S. Levitus, Ed. NOAA Atlas NESDIS 69, U.S. Government Printing Office, Washington, D.C: S. Levitus, Ed. NOAA Atlas NESDIS 69, U.S. Government Printing Office, 2010.

Barcelona Expert Centre Team, "SMOS-BEC ocean and land products description" 2013. http://cp34-bec.cmima.csic.es/doc/BEC-SMOS-0001-PD.pdf

Barcelona Expert Centre Team, "Quality report: Validation of SMOS-BEC products from 2013001 reprocessing campaign, Years 2010-2013 dataset”, 2014. http://cp34bec.cmima.csic.es/doc/BEC-SMOS-0005-QR.pdf

J. Boutin, P. Waldteufel, N. Martin, G. Caudal, and E. Dinnat, "First assessment of SMOS data over open Ocean: Part II- Sea surface salinity” J. Atmos. Ocean. Tech., vol. 21, pp. 1432-1447, 2012.

F.P. Bretherton, E. D. Russ, and C. B. Fandry, "A technique for objective analysis and design of oceanographic experiments applied to MODE-73". Deep-Sea Research, vol. 23, pp. $559-582,1976$.

D.B. Chelton, R. A. deSzoeke, M. G. Schlax, K. El Naggar, and N. Siwertz, "Geographical variability of the first-baroclinic Rossby radius of deformation". J. Phys. Oceanogr., vol. 28, pp. 433-460, 1998.

S.T. Cole, D. L. Rudnick, and John A. Colosi, "Seasonal evolution of upper-ocean horizontal structure and the remnant mixed layer". J. Geophys. Res., vol. 115, C04012, 2010. DOI:10.1029/2009JC005654 
S.T. Cole and D. L. Rudnick, "The spatial distribution and annual cycle of upper ocean thermohaline structure". J. Geophys., Res., vol. 117, C02027, 2012. DOI: 10.1029/2011JC007033.

T. Delcrois, M. J. McPhaden, A. Dessier, and Y. Gouriou, "Time and space scales for sea surface salinity in the tropical oceans". Deep-Sea Res. I, vol. 52, pp. 787-813, 2005.

C.J. Donlon, M. Martin, J. Stark, J. Roberts-Jones, E., Fiedler, and W. Wimmer, "The Operational Sea surface Temperature and Sea Ice Analysis (OSTIA) system”. Remote Sens. Environ., vol. 116, pp. 140-158, 2012.

W.J. Emery and R. E. Thomson, Data analysis methods in physical oceanography, Second and revised edition 2001, Elsevier Science B. V., 2012.

R.M. Errico, R. M. (1985), , "Spectra computed from a limited area grid". Mon. Weather Rev., vol. 113, pp. 1554-1562, 1985.

R. Frehlich and R. Sharman, "The use of structure functions and spectra from numerical model output to determine effective model resolution". Mon. Weather Rev., vol. 136, pp. 1537-1553, 2008. DOI: 10.1175/02007MWR2250.1

F. Gaillard, T. Reynaud, V. Thierry, N. Kolodziejczyk, and K. von Shuckmann, "In situbased reanalysis of the global ocean temperature and salinity with ISAS: Variability of the Heat Content and Steric Height". J. of Clim., vol. 29, pp. 1305-1323, 2016. DOI: 10.1175/JCLI-D-15-0028.1

V. Gonzalez-Gambau, E. Olmedo, J. Martinez, A. Turiel and I. Durán, "New approach for the improvement of SMOS salinity retrievals in coastal regions", IEEE J. Of Selec. Top. in appl. Earth Obs. and Rem. Sens., under review. 
V. Gonzalez-Gambau, A. Turiel, E. Olmedo, J. Martinez, I. Corbella, and A. Camps, "Nodal sampling, a new image reconstruction algorithm for SMOS”. IEEE T. Geosci. Remote, in press, 2016a. DOI: 10.1109/TGRS.2015.2499324.

V. Gonzalez-Gambau, E. Olmedo, A. Turiel, J. Martinez, J. Ballabrera, M. Portabella, and M. Piles, "Enhancing SMOS brightness temperature over the ocean using the nodal sampling image reconstruction technique". Rem. Sens. Environ., in press, 2016b. DOI: 10.1016/j.rse.2015.12.032

J. Gourrion, Sabia, R., Portabella, M., Tenerelli, J., Guimbard, S., Camps, A., "Characterization of the SMOS instrumental error pattern correction over the ocean". $\begin{array}{llllllll}\text { IEEE Geosci. Rem. Lett., vol. } 9 & \text { (4), pp. 793-797, } 2012 .\end{array}$ DOI:10.1109/LGRS.2011.2181990, 2012.

J. Gourrion, S. Guimbard, M. Portabella and R. Sabia, "Towards an optimal estimation of the SMOS antenna-frame systematic errors". IEEE T. Geosci. Remote, vol. 51 (9), pp. $4752-$ 4760, 2013. DOI:10.1109/TGRS.2013.2271593.

O. Hernandez, J. Boutin, N. Kolodziejcyk, G. Reverdin, N. Martin, F. Gaillard, N. Reul, and J. L. Vergely, "SMOS salinity in the subtropical North Atlantic salinity maximum: 1. Comparison with Aquarius and in situ salinity”. J. Geophys. Res., vol. 119, pp. 8878-8896, 2014. DOI: $10.1002 / 2013 J C 009610$.

N. Hoareau, M. Umbert, J. Martinez, A. Turiel, and J. Ballabrera-Poy, "On the potential of data assimilation to generate SMOS-Level 4 of sea surface salinity". Rem. Sens. Environ., vol. 146, pp. 188-200, 2014.

G. Jorda, D. Gomis, and M. Talone, "The SMOS L3 mapping algorithm for sea surface salinity”. IEEE Trans. Geosci. Remote Sens., vol. 49(3), pp. 1032-105, 2011. 
N. Kolodziejczyk, J. Boutin, J. Vergely, S. Marchand, N. Martin and G. Reverdin, "Mitigation of systematic errors in SMOS sea surface salinity". Rem. Sens. of Environ., vol. 180, pp. 164-177, 2016.

N. Kolodziejczyk, O. Hernandez, J. Boutin, and G. Reverdin, "SMOS salinity in the subtropical North Atlantic salinity maximum: 2. Two-dimensional horizontal thermohaline variability”. J. Geophys. Res., vol. 120, pp. 972-987, 2015a. DOI: 10.1002/2014JC010103.

N. Kolodziejczyk, G. Reverdin, J. Boutin, and O. Hernandez, "Observation of the surface horizontal thermohaline variability at mesoscale to submesoscale in the north-eastern subtropical Atlantic Ocean”. J. Geophys. Res., vol. 120, pp. 2588-2600, 2015b. DOI: 10.1002/2014JC010455.

G.S.E. Lagerloef, Satellite measurements of Salinity, In: Steele, J., Thorpe S., Turekian K. (eds.) Encyclopedia of Ocean Sciences, Academic Press, pp. 2511-2516, 2001.

G. Lagerloef, H.-Y. Kao, T. Meissner, and J. Vazquez, “Aquarius salinity validation analysis; Data Version 4.0”, 2015. Available at ftp://podaac-ftp.jpl.nasa.gov.

R. A. Locarnini, A. V. Mishonov, J. I. Antonov, T. P. Boyer, H. E. Garcia, O. K. Baranova, M. M. Zweng, and D. R. Johnson, “World Ocean Atlas 2009, Volume 1: Temperature”. S. Levitus, Ed., NOAA Atlas NESDIS 68, U.S. Government Printing Office, Washington, D.C., 184 pp., 2010.

R. A. Locarnini, A. V. Mishonov, J. I. Antonov, T. P. Boyer, H. E. Garcia, O. K. Baranova, M. M. Zweng, C. R. Paver, J. R. Reagan, D. R. Johnson, M. Hamilton, D. Seidov, "World Ocean Atlas 2013, Volume 1: Temperature”. S. Levitus, Ed.; A. Mishonov, Technical Ed.; NOAA Atlas NESDIS 73, 40 pp.,2013. 
M. Madec and NEMO team, NEMO ocean engine, version 3.4, Note du Pôle de modélisation de l'Institut Pierre-Simon Laplace, No 27, ISSN No 1288-1619, 2012.

S. Mecklenburg, M. Drush, Y. H. Kerr, J. Font, M. Martin-Neira, S. Delwart, G. Buenadicha, N. Reul, E. Daganzo-Eusebio, R. Oliva, and R. Crapolicchio, "ESA's Soil Moisture and Ocean Salinity Mission: Mission performance and Operations”. IEEE T. Geosci. Remote, vol. 50, pp. 1354-1366, 2012. DOI: 10.1109/TGRS.2012.2187666.

J. Mignot and C. Frankignoul, "On the interannual variability of surface salinity in the Atlantic”. Clim. Dyn., vol. 20, pp. 555-565, 2003.

A. Montuori, M. Portabella, S. Guimbard, C. Gabarró, and M. Migliaccio, “Assessment of the SMOS inversion scheme for salinity and wind speed retrieval purposes". Eur. J. Remote Sensing, vol. 46, pp. 855-873, 2013. DOI: 10.5721/EuJRS20134651.

V. Nieves, C. Llebot, A. Turiel, J. Solé, E. García-Ladona, and M. Estrada, "Common turbulent signature in sea surface temperature and chlorophyll maps”. Geophys. Res. Lett., vol. 34, L23602, 2007.

E. Olmedo, J. Martínez, M. Umbert, N. Hoareau, M. Portabella, J. Ballabrera-Poy and A. Turiel, "Improving time and space resolution of SMOS salinity maps using multifractal fusion”. Rem. Sens. Environ., vol. 180, pp. 246-263, 2016.

L. Parent, N. Ferry, B. Barnier, G. Garric, C. Bricaud, C-E Testut, O. Le Galloudec, J-M Lellouche, E. Greiner, M. Drevillon, E. Rémy, J-M Molines, S. Guinehut, and C. Cabanes “GLOBAL eddy-permitting ocean reanalysis and simulations of the period 1992 to present", Venice, Italy: Proceeding of the "20 years of progress in radar altimetry", 24-29 September, 2012. 
D. B. Percival and A. T. Walden Spectral analysis for physical applications, Cambridge University Press, Cambridge, New York, USA, 1993.

O. Pont, A. Turiel and H. Yahia, " Singularity analysis of digital signals through the evaluation of their unpredictable point manifold", International Journal of Computer Mathematics, vol. 90(8), pp. 1693-1707, 2013. DOI: 10.1080/00207160.2012.748895

M. Portabella and A. C. M. Stoffelen, "On scatterometer Ocean Stress". J. Atm. Ocean. Tech., vol. 26(2), pp. 368-382, 2009. DOI: 10.1175/2008JTECHO578.1

W. H. Press, B. P. Flannery, S. A. Teukolsky, and W. T. Vetterling, Numerical recipes, Cambridge University Press, Cambridge, New York, USA, 1989.

N. Reul, J. Terenelli, J. Boutin, B. Chapron, F. Paul, E. Brion, et al., "Overview of the First SMOS sea surface salinity products. Part I: Quality assessment for the second half of 2010”. IEEE T. Geosci. Remote, vol. 50(5), pp. 1636-1647, 2012.

G. Reverdin, E. Kestenare, C. Frankignoul, and T. Delcroix. "Surface salinity in the Atlantic Ocean (30S-50N)". Prog. Oceanogr., vol. 73, pp. 311-340, 2007.

R. W. Reynolds and, D. B. Chelton, "Comparisons of daily sea surface temperature analyses for 2007-2008”. J. Climate, vol. 23, pp. 3545-3562, 2010.

W. Rubin, Real and complexe analysis. Mc Graw Hill, New York, USA, 1987.

SO-TN-IDR-GS-0006, "SMOS Level 2 and Auxiliary Data Products Specifications SO-TNIDR-GS-0006”, INDRA, Issue 8 revision 4, 2016.

A. Stoffelen, "Error modeling and calibration: towards the true surface wind speed". $J$. Geophys. Res., vol. 103, pp. 7755-7766, 1998. DOI: 10.1175/2010JCLI3294.1. 
A. H. Taylor and J. A. Stephens, "Seasonal and year to year variations in surface salinity at nine North Atlantic Ocean weather stations". Ocean. Acta, vol. 3(4), pp. 421-430, 1980.

A. Turiel, J. Isern-Fontanet, E. García-Ladona, and J. Font, "Multifractal method for the instantaneous evaluation of the stream function in geophysical flows". Physical Review Letters, vol. 95, pp. 104502, 2005.

A. Turiel, C. J. Pérez-Vicente and J. Grazzini, "Numerical methods for the estimation of multifractal singularity spectra on sampled data: a comparative study". J. of Comput. Phys., vol. 216, pp. 362-390, 2006.

A. Turiel, J. Solé, V. Nieves, J. Ballabrera-Poy and E. García-Ladona, “Tracking oceanic currents by singularity analysis of micro-wave sea surface temperature images". Rem. Sens. Environ., vol. 112, pp. 2246-2260, 2008a.

A. Turiel, H. Yahia and C. J. Pérez-Vicente, "Microcanonical multifractal formalism: A geometrical approach to multifractal systems, Part I: Singularity analysis”. Journal of Physics A, vol. 41, pp. 015501, 2008b. DOI: 10.1088/1751-8113/41/1/015501.

A. Turiel, V. Nieves, E. García-Ladona, J. Font, M. H. Rio and G. Larnicol, “The multifractal structure of satellite sea surface temperature maps can be used to obtain global maps of streamlines”. Ocean Science, vol. 5, pp. 447-460, 2009.

M. Umbert, N. Hoareau, A. Turiel, and J. Ballabrera-Poy, "New blending algorithm to synergize ocean variables: The case of SMOS sea surface salinity maps". Rem. Sens. Environ., vol. 146, pp. 172-187, 2014.

UNESCO, "The Practical Salinity Scale 1978 and the International Equation of State of Seawater 1980," United Nations Educational, Scientific and Cultural Organization, Paris, 
France, UNESCO Technical Papers in Marine Science Number 36, 10th report of the Joint Panel on Oceanographic Tables and Standards, 1981.

J. Vogelzang,,A. Stoffelen, A. Verhoef, and J. Figa-Saldaña, “On the quality of highresolution scatterometer winds". J. Geophys. Res., vol. 116, C10033, 2011. doi: 10.1029/2010JC006640.

J. Vogelzang, "How to calculate wind spectra", Document NWPSAF-KN-TR-008, version 1.1, March, 2013.

J. Vogelzang, G.P. King, and A. Stoffelen, "Spatial variances of wind fields and their relation to second-order structure functions and spectra". J. Geophys. Res., vol. 120, pp. 10481064, 2015. DOI: 10.1002/2014JC010239.

S. Yueh, W. Tang, A. Fore, and A. Hayashi, Aquarius CAP Algorithm and Data User Guide, June, 2014. ftp://podaac-ftp.jpl.nasa.gov/allData/aquarius/docs/CAPv3/Aquarius-CAP- UserGuide-v3.0.pdf

M. M. Zweng, J.R. Reagan, J.I. Antonov, R.A. Locarnini, A.V. Mishonov, T.P. Boyer, H.E. Garcia, O.K. Baranova, D.R. Johnson, D. Seidov and M.M. Biddle, "World Ocean Atlas 2013, Volume 2: Salinity". S. Levitus, Ed., A. Mishonov Technical Ed.; NOAA Atlas NESDIS 74, 39 pp., 2013. 


\section{Figures:}

Fig. 1: Regions under study Spatial distribution of Rossby radii derived by Chelton et al. (1998). Blue boxes represent the regions under study: NATL $\left(0^{\circ}-64^{\circ} \mathrm{N}\right.$ and $\left.27^{\circ}-33^{\circ} \mathrm{W}\right)$; ITCZ $\left(4^{\circ}-10^{\circ} \mathrm{N}\right.$ and $\left.96^{\circ}-160^{\circ} \mathrm{W}\right)$; SPURS $\left(22^{\circ}-28^{\circ} \mathrm{N}\right.$ and $\left.28^{\circ}-60^{\circ} \mathrm{W}\right)$; ARC $\left(39^{\circ}-45^{\circ} \mathrm{S}\right.$ and $30^{\circ}-$ $\left.94^{\circ} \mathrm{E}\right)$; STP $\left(27^{\circ}-33^{\circ} \mathrm{S}\right.$ and $\left.106^{\circ}-170^{\circ} \mathrm{W}\right)$ and $\operatorname{SPAC}\left(14^{\circ} \mathrm{N}-50^{\circ} \mathrm{S}\right.$ and $\left.122^{\circ}-128^{\circ} \mathrm{W}\right)$.

Fig. 2: Annual SSS mean: 2012. a) SL3, b) SOI, c) SL4, d) S13, e) S09, f) Aq, g) SC, h) SG and i) AqC monthly products.

Fig. 3: Intra-annual SSS variance: 2012. a) SL3, b) SOI, c) SL4, d) S13, e) S09, f) Aq, g) SC, h) SG and i) AqC monthly products.

Fig. 4: OSTIA SST singularity exponents: January 2012. Maps of singularity exponents derived from January 2012 OSTIA SST (TO) map.

Fig. 5: Singularity exponent of SSS: January 2012. Singularity exponent maps of the following monthly SSS products for January 2012: a) SL3, b) SOI, c) SL4, d) S13, e) S09, f) Aq, g) SC, h) SG and i) AqC.

Fig. 6: Condition histogram. Histograms of SSS singularity exponents conditioned by the value of the corresponding SST singularity exponent values, for each SSS product for January 2012: a) SL3, b) SOI, c) SL4, d) S13, e) S09, f) Aq, g) SC, h) SG and i) AqC. 
Fig. 7: Wavenumber spectra comparison. Power density spectra (PDS) (left) and singularity power spectra (SPS) (right) in STP region for six selected noised SSS maps in addition to the original. White noises of different amplitudes have been added to the same SG snapshot corresponding to June 2013 (black line). In total, ten new maps were created from the original SSS map by adding white noise with standard deviations ranging from 0.10 to 1.00 in steps of 0.10 . The six selected noised SSS spectra are from white noise with a standard deviation of 0.10 in magenta, 0.20 in blue, 0.30 in green, 0.50 in cyan, 0.70 in yellow and 1.00 value in red. A grey line, representing slope of -2.4 , is provided for reference.

Fig. 8: OSTIA SST Singularity power spectra: 2012. Singularity power spectra (SPS) of OSTIA SST for 2012 in the following ocean regions: ARC (red line), ITCZ (black), NATL (magenta), SPAC (blue) STP (cyan) and SPURS (Green). A -2.4 slope reference line (grey) is shown for reference. The 95\% confidence interval is calculated as in Emery and Thomson [2001]. The number of degrees of freedom used for the SPS estimation depends on the resolution of the product, i.e., 168, 312 and 600 for the products of one, half and a quarter degree, respectively.

Fig. 9: SSS Singularity power spectra: 2012. Same as Figure 8 but for the singularity exponents maps of the following SSS products: a) SL3, b) SOI, c) SL4, d) S13, e) S09, f) Aq, g) SC, h) SG and i) AqC

Fig. 10: Wavenumber spectral slope comparison. Singular power spectra (SPS) slopes (left) and PDS slopes (right) values, calculated for the intermediate scales (wavelength range of 400-800 km), for each salinity product, in each of the six ocean regions (see colour legend) 
for 2012, as well as for the five SST products. The error bars represent to the estimated standard deviation for each slope. The nine SSS product slopes are shown: SL3, SOI, SL4, Aq, AqC, SG, SC, S09, and S13; The reference OSTIA SST (TO) and four SST products: GLORYS2V3 SST (TG) from the numerical simulation, CORA SST (TC) from the in situ reanalysis, and the two climatologies from WOA 2009 SST (T09) and WOA 2013 SST (T13) 


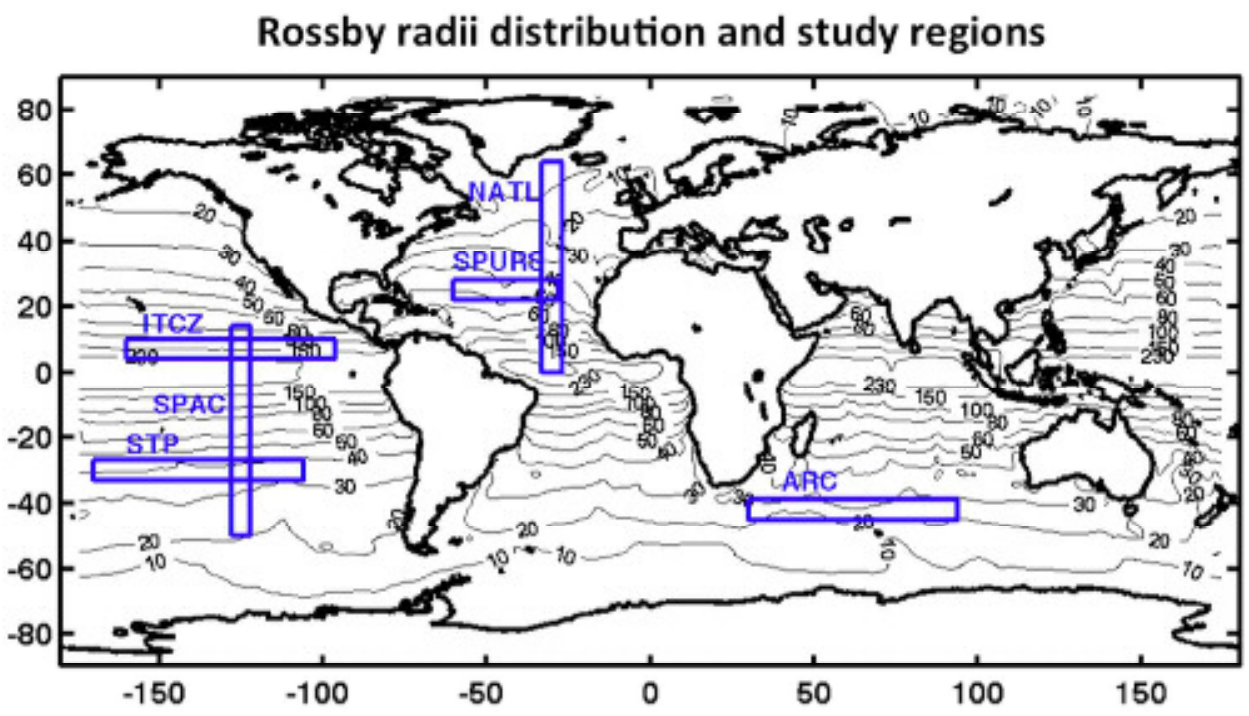




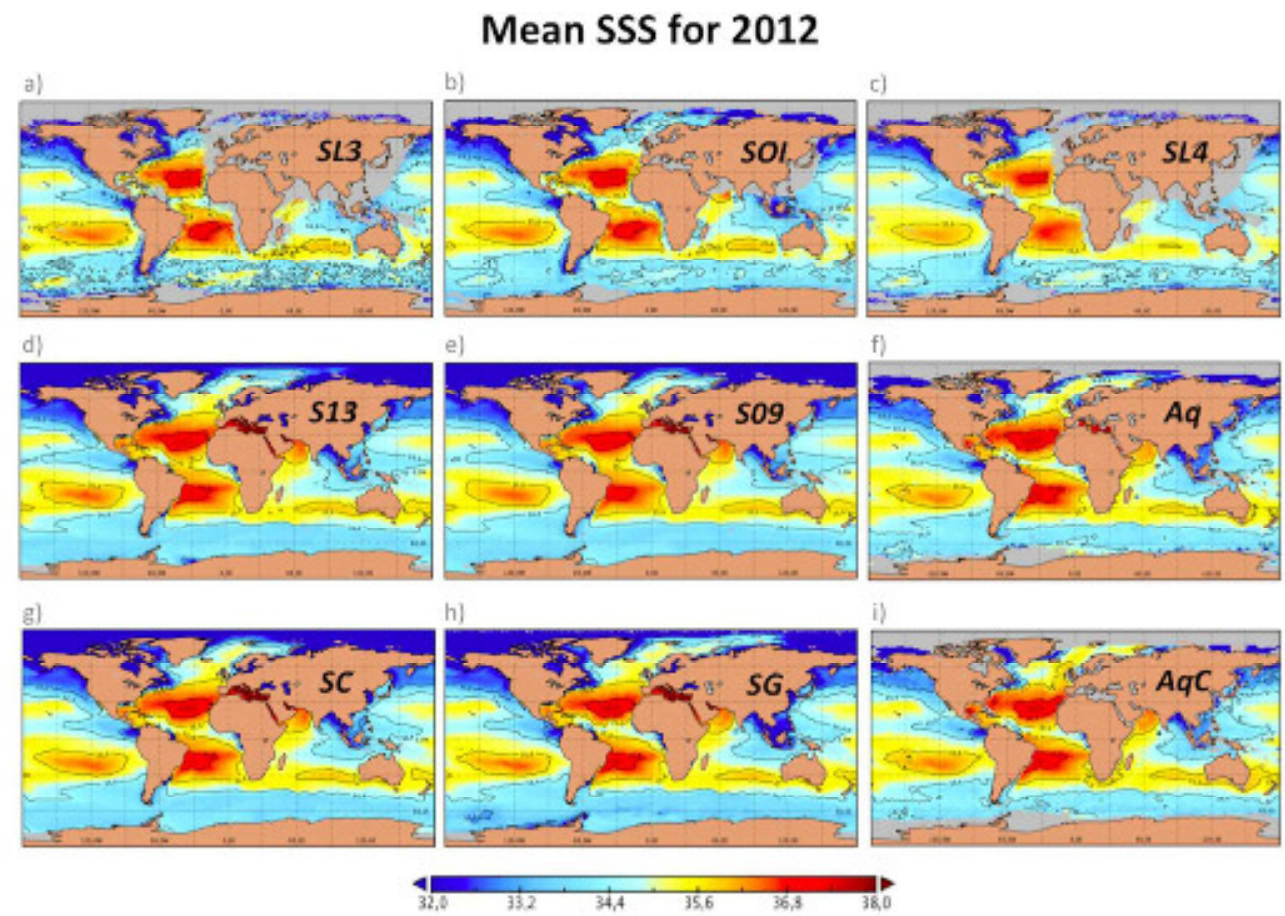




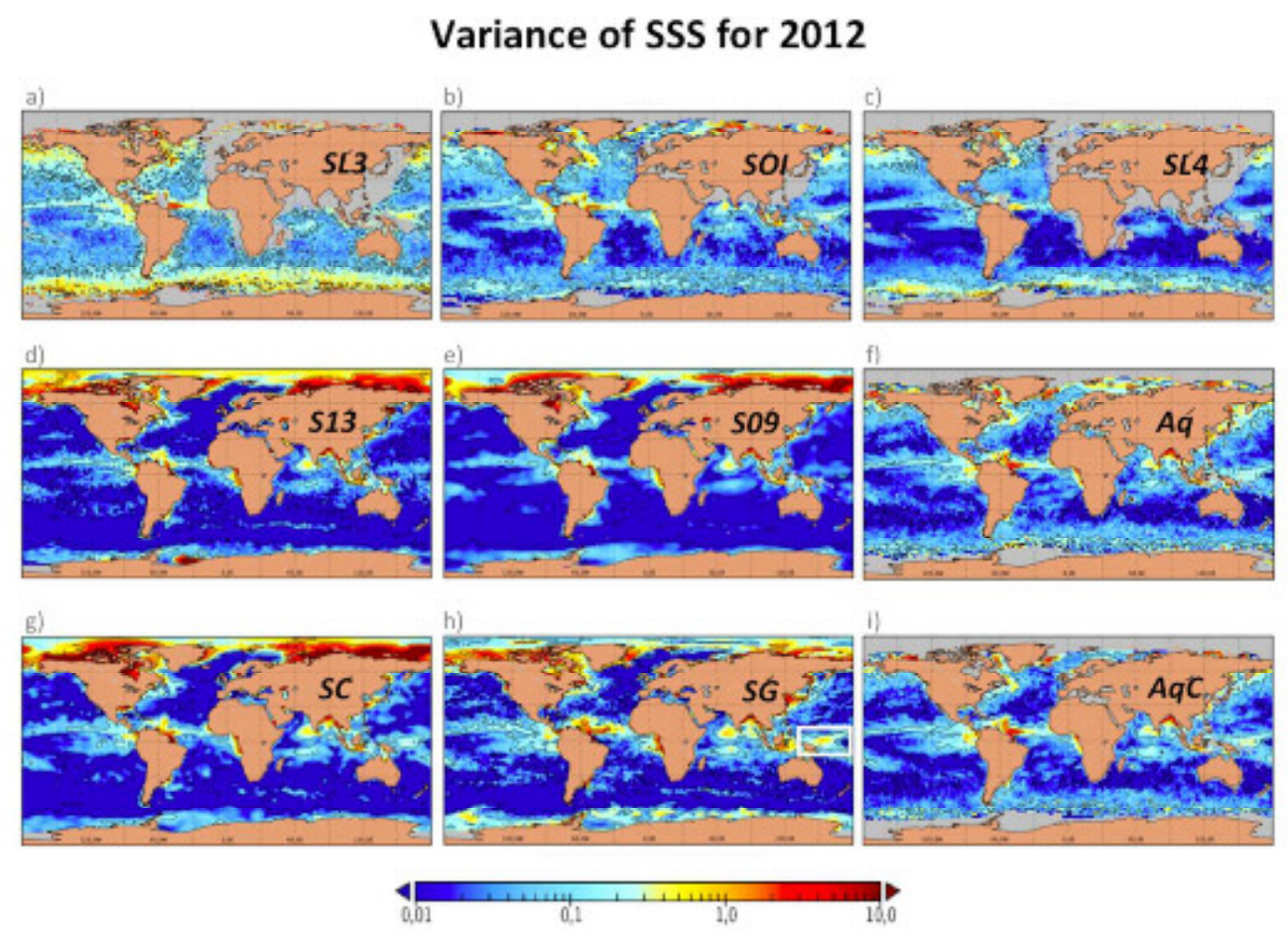


Singularity exponents of OSTIA SST - January 2012

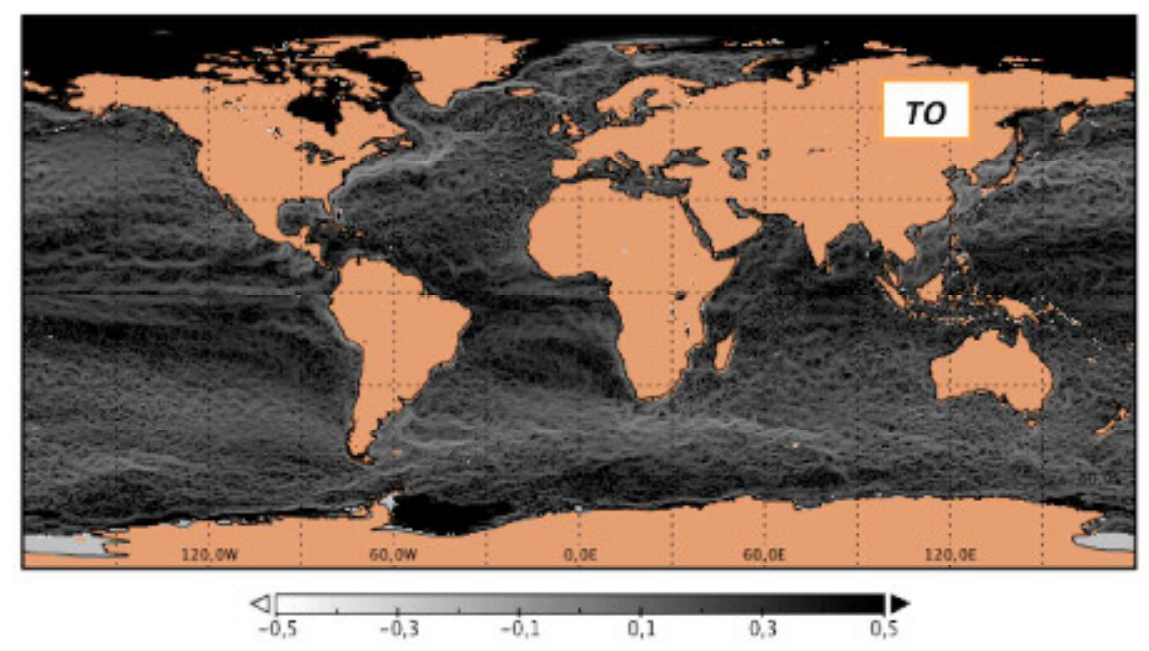




\section{Singularity exponents of SSS - January 2012}
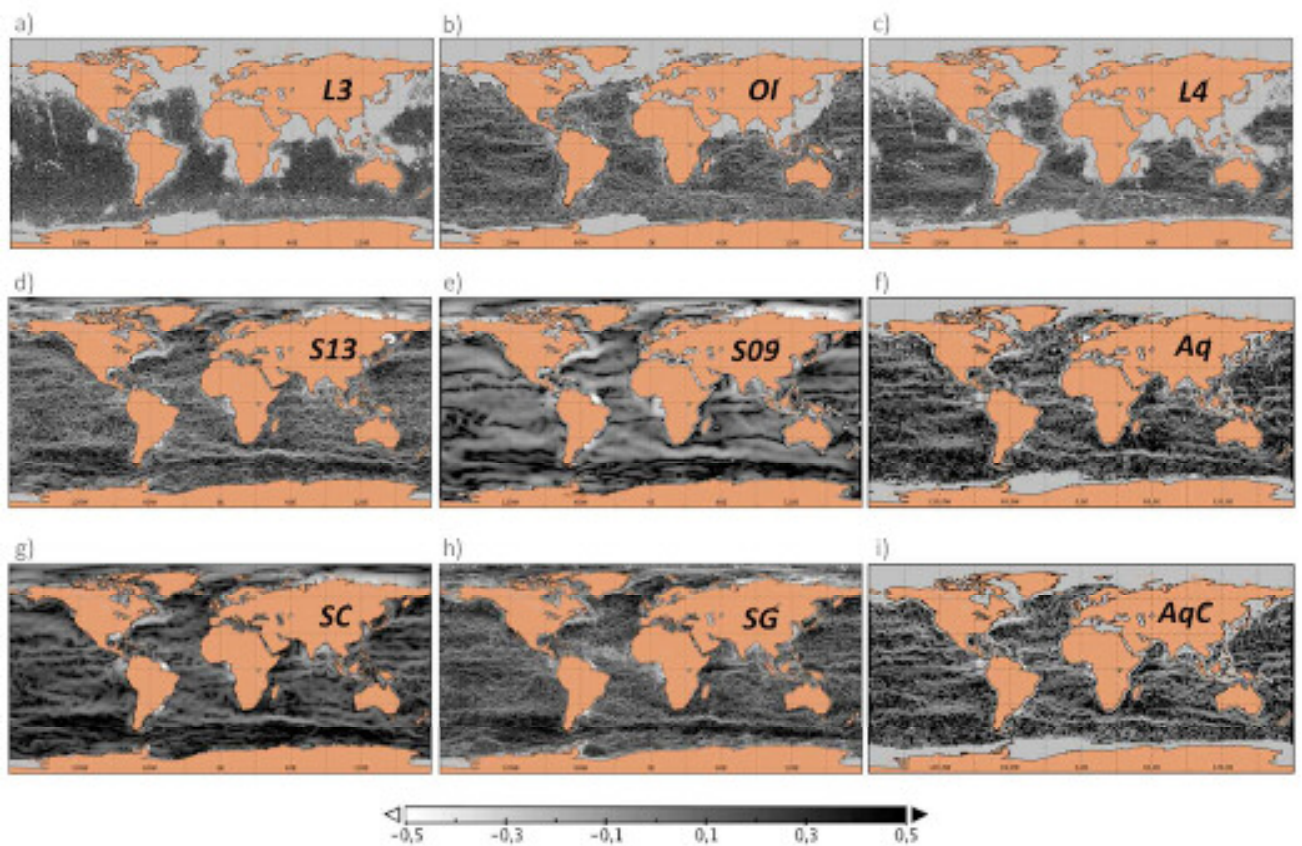

30

31

32 


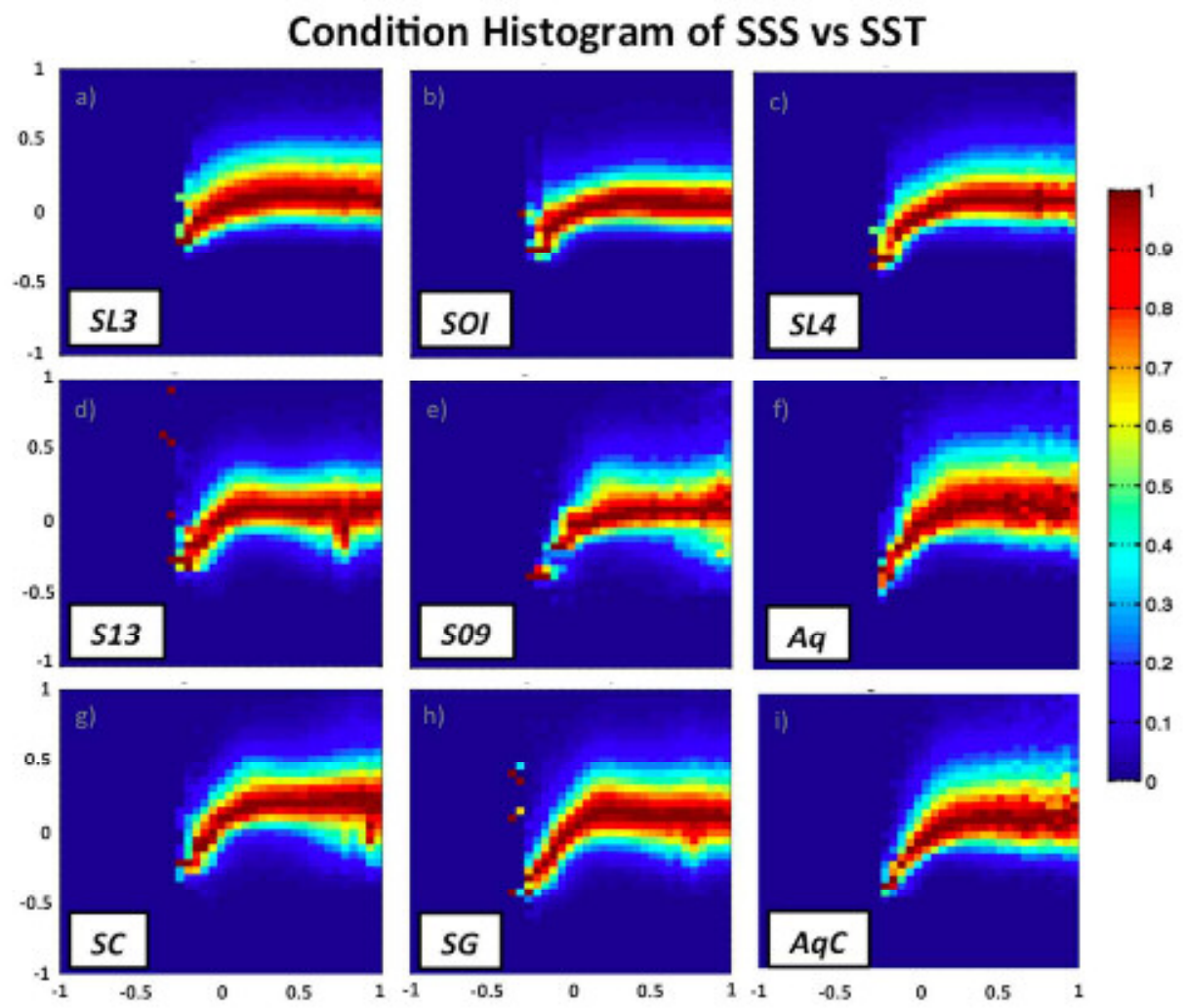




\section{Effect of white noise in the PDS and the SPS}
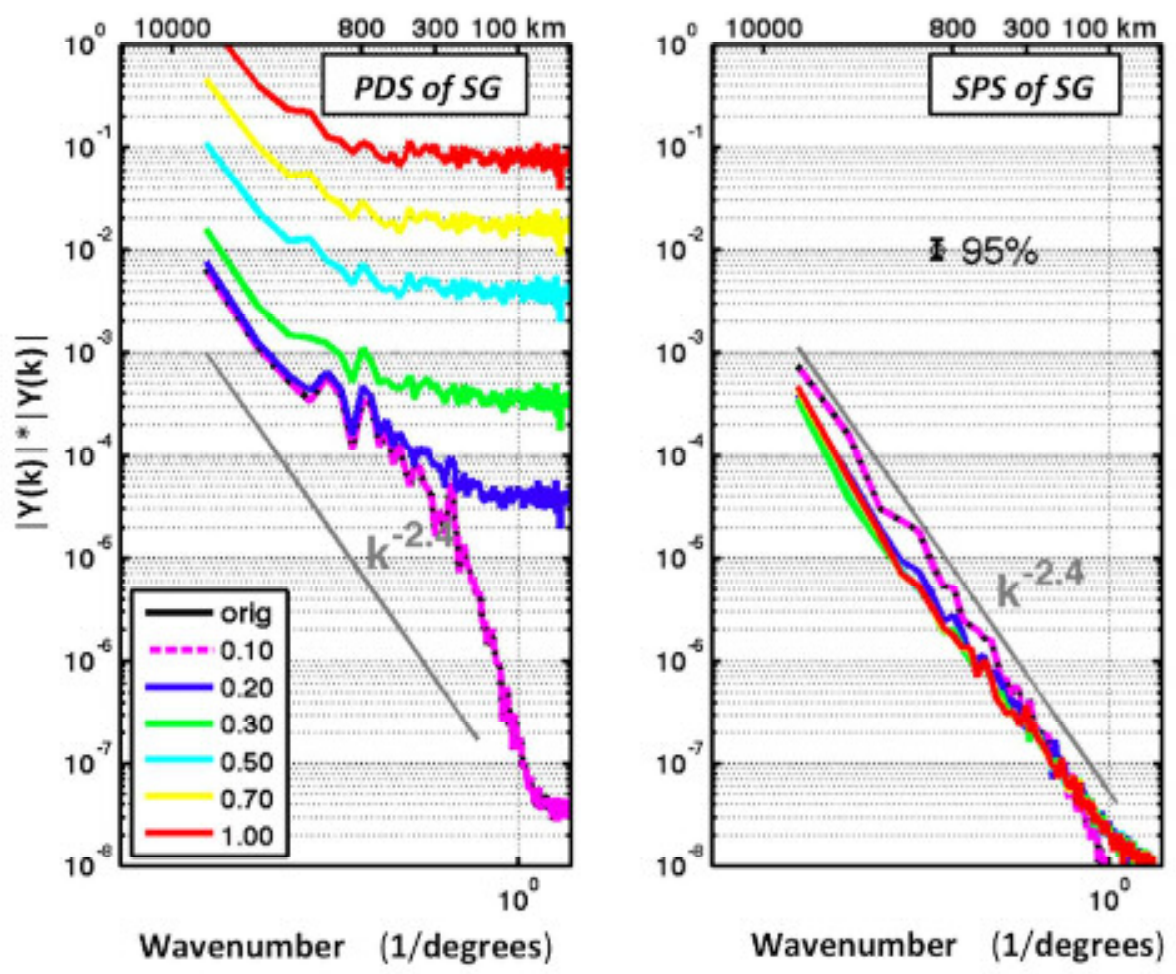


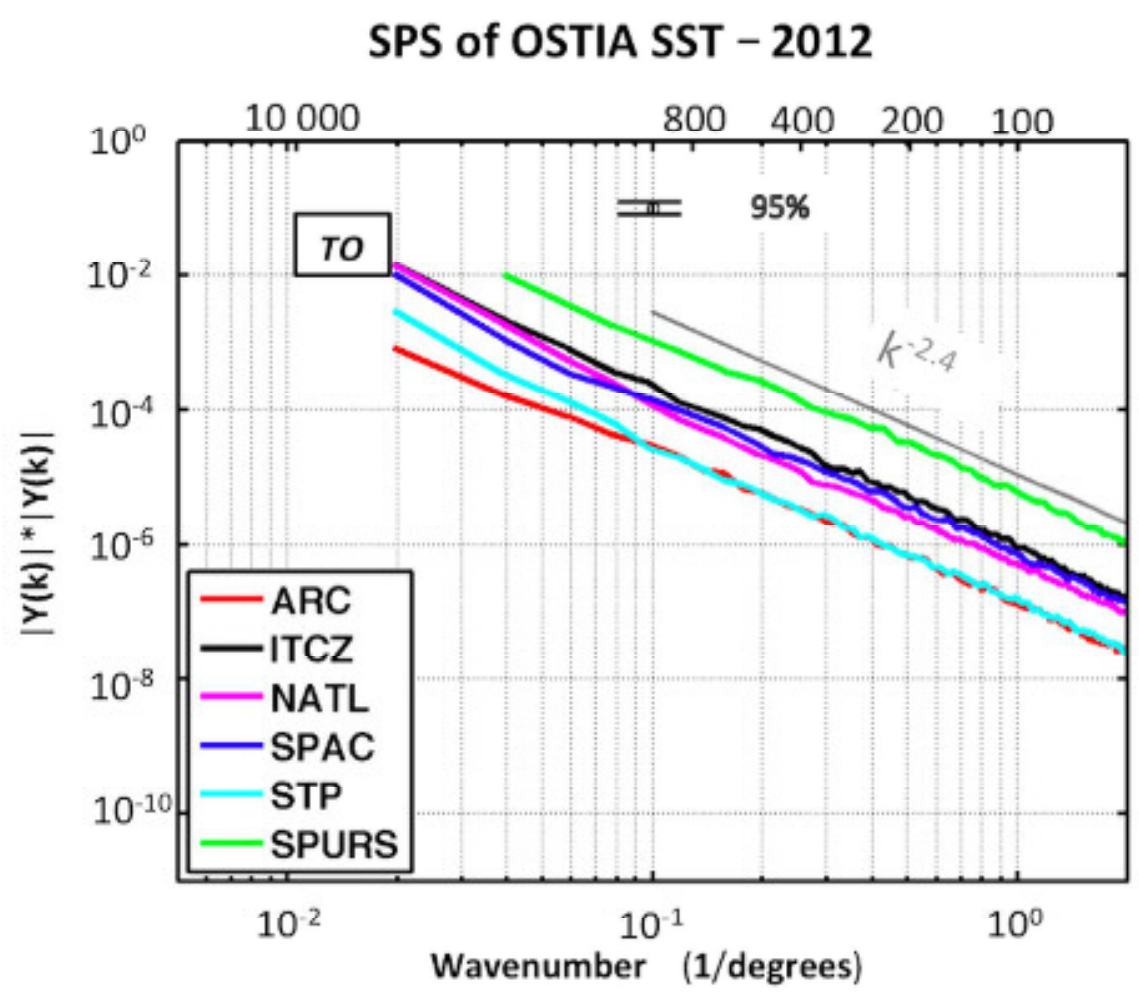




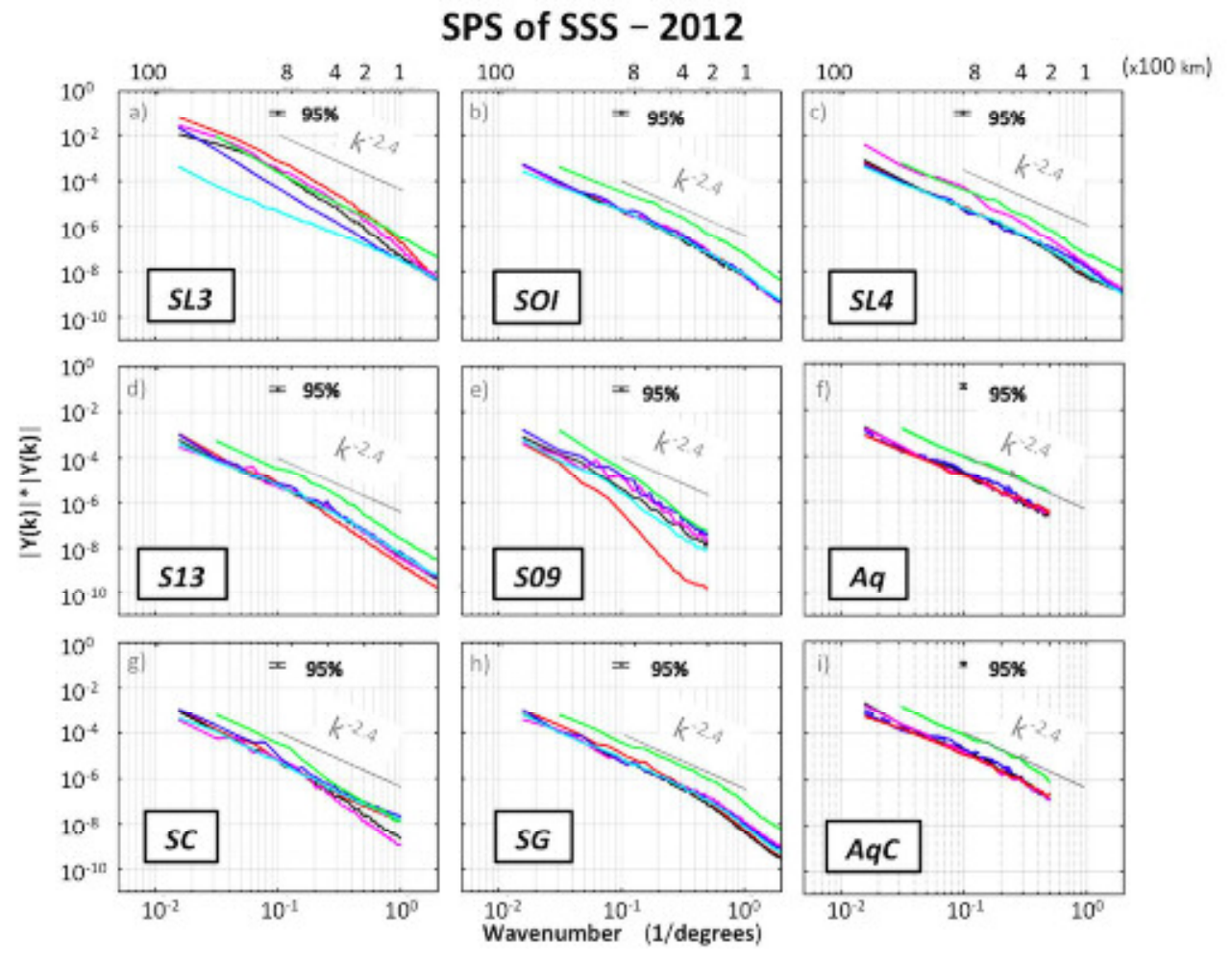


Page 77 of 77

Transactions on Geoscience and Remote Sensing

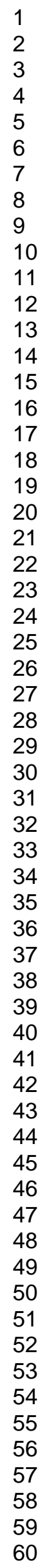

